\title{
Genome-wide DNA methylation and RNA expression differences correlate with invasiveness in melanoma cell lines
}

\author{
Jyoti Motwani**,1(D), Euan J Rodger ${ }^{1,2}$ (D), Peter A Stockwell ${ }^{1}$, Bruce C Baguley ${ }^{2,3}$ (D), Erin C \\ Macaulay ${ }^{1}$ (D) \& Michael R Eccles*,1,2 (iD \\ ${ }^{1}$ Department of Pathology, Otago Medical School - Dunedin Campus, University of Otago, Dunedin 9054, New Zealand \\ ${ }^{2}$ Maurice Wilkins Centre for Molecular Biodiscovery, Level 2, 3A Symonds Street, Auckland 1010, New Zealand \\ ${ }^{3}$ Auckland Cancer Society Research Centre, The University of Auckland, Auckland 1023, New Zealand \\ *Author for correspondence: Tel.: +64 3479 7878; michael.eccles@otago.ac.nz \\ **Author for correspondence: mail.jyotim@gmail.com
}

\begin{abstract}
Aims \& objectives: The aim of this study was to investigate the role of DNA methylation in invasiveness in melanoma cells. Materials \& methods: The authors carried out genome-wide transcriptome (RNA sequencing) and reduced representation bisulfite sequencing methylome profiling between noninvasive $(n=4)$ and invasive melanoma cell lines $(n=5)$. Results: The integration of differentially expressed genes and differentially methylated fragments (DMFs) identified 12 DMFs (two in AVPI1, one in HMG2OB, two in $B C L 3$, one in NTSR1, one in SYNJ2, one in ROBO2 and four in HORMAD2) that overlapped with either differentially expressed genes (eight DMFs and six genes) or cis-targets of IncRNAs (five DMFs associated with cis-targets and four differentially expressed IncRNAs). Conclusions: DNA methylation changes are associated with a number of transcriptional differences observed in noninvasive and invasive phenotypes in melanoma.
\end{abstract}

First draft submitted: 4 December 2020; Accepted for publication: 24 February 2021; Published online: 30 March 2021

Keywords: cell invasion $\bullet$ DNA methylation $\bullet$ gene expression $\bullet$ global methylation $\bullet$ invasive $\bullet$ IncRNAs $\bullet$ metastatic melanoma $\bullet$ noninvasive $\bullet$ phenotype switching $\bullet$ RNA-seq

Melanoma, derived from pigment-producing cells called melanocytes, is the most serious form of skin cancer. New Zealand has among the highest rates of melanoma in the world, with an incidence in the population exceeding 50 per 100,000, making melanoma the fourth most common cancer type in New Zealand [1]. Melanoma patients have a 5-year survival rate greater than $95 \%$ if the Breslow depth of the melanoma is less than $1 \mathrm{~mm}$ following early detection and excision. However, melanoma metastasis occurs in $15-25 \%$ of patients whose tumor has a Breslow depth between 1 and $4 \mathrm{~mm}$ [2]. Intrinsic molecular changes that drive invasiveness in melanoma are not well understood, although enhanced invasive behavior of melanoma cells is thought to occur as an important step during the acquisition of the metastatic phenotype.

The authors have previously described a panel of New Zealand melanoma (NZM) cell lines derived from metastatic melanomas of NZM patients [3]. Unintuitively, metastatic melanoma cell lines are frequently noninvasive. These NZM cell lines exhibit diverse phenotypes and invasive behaviors, and extensive molecular characterization of the NZM lines has revealed that they closely match the spectrum of molecular alterations observed in melanomas analyzed from patients in the New Zealand population [4]. Analysis of the invasiveness of NZM cell lines indicates that, as has been observed in other melanoma cell line panels [5], the NZM cell lines can be grouped into invasive and minimally invasive to noninvasive subgroups, with a characteristic gene expression signature corresponding to each subgroup [6].

Studies have revealed that development of cancer is not solely dependent on changes in protein-coding genes; the noncoding genome is also emerging as a regulatory player in tumorigenesis [7]. The noncoding genome involves genomic elements such as enhancers, insulators, alternative promoters, repeat elements and regions that 
are transcribed as ncRNAs. About two-thirds of the human genome comprises repeat elements, which are classified into two main categories: transposable elements and tandem repeats. Transposable elements represent about $45 \%$ of the mammalian genome and are classified as retrotransposons (class I) and DNA transposons (class II). Retrotransposons are further classified into long terminal repeats (LTRs) and non-LTR retrotransposons. LTRs are repeat sequences formed by reverse transcription of RNA that is derived from retroviruses. LTRs and endogenous retroviruses (ERVs) mediate integration of retroviral DNA into the host genome. They comprise up to $8 \%$ of the genome and are characterized by LTRs at $5^{\prime}$ and $3^{\prime}$ ends flanking the sequence that encodes a minimum of two proteins, gag and pol. ERVs consist of gag, pol and env viral proteins. ERVs are mainly classified as class I (ERV-H), class II (ERV-K) and class III (ERV-L, ERV-S). By contrast, non-LTRs include long interspersed nuclear elements (LINEs), short interspersed nuclear elements (SINEs) and SINE-VNTR-Alus, elements that contain a SINE region, tandem repeats and an Alu-like sequence, which represent about one-third of the human genome [8]. The tandem repeats include minisatellite, microsatellite and satellite DNA.

Growing evidence suggests a role for repeat elements in various cancers. In normal somatic cells, repeat elements are usually repressed to maintain genomic integrity. The primary mechanism regulating repeat element expression is epigenetic; namely, DNA methylation and histone modifications (H3K9 methylation), which act together to suppress repeat elements [9]. Interestingly, repeat elements are expressed in cancer cells. The global DNA hypomethylation in cancer cells is known to affect the intergenic and intronic regions, in particular the repeat elements, and is associated with genomic instability and increased mutation events through the activation of repeat elements [10]. LINE-1 promoter hypomethylation is reported in bladder cancer [11], chronic myeloid leukemia [12], hepatocellular carcinoma [13], prostate cancer [14] and melanoma [15]. The transcriptional activity of LTRs (ERVs) is regulated by DNA methylation [16], and the expression of ERV transcripts is well known in melanoma [17], breast cancer [18], ovarian cancer [19], colorectal cancer [20], prostate cancer [21], hepatocellular carcinoma [22], renal carcinoma [23] and endometrial carcinoma [24]. Furthermore, recent studies have explored an association between expression of repeat elements and poor patient survival and immunosuppressive phenotypes in cancer [25].

In addition to repeat elements, recent studies have reported the role of lncRNAs in cancer. IncRNAs can epigenetically control gene expression by interacting with chromatin modifiers and transcription factors. They can regulate DNA methylation by interacting directly or indirectly with DNA methyltransferases [26]. About $80 \%$ of lncRNAs overlap with repeat elements. The majority of the overlapping repeat elements are LTRs (ERVs), whereas the minority are LINEs and SINEs [27]. Repeat element insertions within lncRNAs serve as regulatory sites for cell proliferation. Hence, the deregulation of repeat elements embedded within lncRNAs disrupts their function and is associated with carcinogenesis.

Previous studies have analyzed genome-wide DNA methylation in gene-coding regions to investigate mechanisms that regulate phenotype switching between invasive and noninvasive subtypes of cutaneous melanoma [28,29]. However, the role of global DNA methylation changes involving the noncoding genome in melanoma phenotype switching remains unexplored. In this study, the authors present a genome-wide landscape analysis of DNA methylation, gene expression and lncRNA expression in melanoma cell line subgroups characterized as noninvasive and invasive [6,30]. As these two behavioral phenotypes are reversible and independent of tumor stage and mutational status, the authors hypothesized that epigenetic mechanisms play a role in regulating the invasive phenotype. To this end, the authors identified differentially expressed transcripts associated with the invasive phenotype, including expression of various protein-coding genes, IncRNAs and pseudogenes. To further investigate the relationship between RNA expression and DNA methylation, the authors profiled methylomes of noninvasive and invasive cell lines and observed global hypomethylation of ERV elements in the invasive group, most of which resulted from intergenic hypomethylation. In addition, the authors identified differentially methylated loci associated with these two phenotypes as well as differentially methylated and differentially expressed protein-coding and noncoding genes.

\section{Methods}

\section{Cell lines}

All of the cell lines used in this study were human cell lines and included NZM cell lines NZM6, NZM9, NZM11, NZM12, NZM15, NZM22, NZM40 and NZM42 and SK-MEL-28. For comparison of expression with normal melanocytes, HemN-LP and HemA-LP cell lines were used. The NZM cell lines were used at a low passage number and were derived from metastatic melanoma patients from New Zealand. The lines were authenticated and generously provided by B Baguley from the University of Auckland, Auckland, New Zealand, in May 2015. 
SK-MEL-28 was originally purchased from American Type Culture Collection (VA, USA). The NZM cell lines and Skemel28 were cultured in a low-oxygen tissue culture incubator (CB 60; BINDER, NY, USA) that was set at $5 \% \mathrm{O}_{2}$ at $37^{\circ} \mathrm{C}$ with a humidified atmosphere of $5 \% \mathrm{CO}_{2}$. Cells were subcultured when the confluence reached $80-90 \%$. The NZM cell lines were cultured in Minimum Essential Medium Eagle - alpha modification [ $\alpha$-MEM] (12561056; Gibco, Life Technologies, NY, USA) supplemented with 5\% fetal bovine serum (FBS) and $0.1 \%$ insulin-transferrin-sodium selenite supplement (11074547001; Sigma-Aldrich, MO, USA). SK-MEL-28 was cultured in Dulbecco's modified Eagle medium (11995-065; Gibco) supplemented with 10\% FBS. HemN-LP was cultured in Medium 254 (M254500; Gibco) and 0.1\% human melanocyte growth supplement (S0025; Gibco). HemA-LP was cultured in Medium 254 (M254500; Gibco) and 0.1\% human melanocyte growth supplement 2 (S0165; Gibco). The cell lines used in this study were generated from metastatic melanoma after written consent was obtained from all patients under Auckland Area Health Board Ethics Committee guidelines as previously described [3].

\section{Validation of phenotype of cell lines}

The noninvasive and invasive phenotype was determined using precoated $8.0-\mu \mathrm{m}$ Matrigel invasion chambers (354480; Corning, NY, USA) and migration inserts (FAL353097; Corning). The invasion inserts were hydrated by following the manufacturer's instructions. Briefly, Matrigel inserts were placed into a 24-well companion plate (353504; Corning) at room temperature to thaw for $30 \mathrm{~min}$. Next, prewarmed serum-free medium was added to the upper and lower chambers of the insert to hydrate the Matrigel for $2 \mathrm{~h}$ at $37^{\circ} \mathrm{C}$ in the tissue culture incubator. Migration inserts do not require hydration and so were directly used to seed the cells. After invasion chamber hydration, cells were trypsinized, counted and resuspended in $2.5 \mathrm{ml}$ medium with no FBS to obtain 125,000 cells, and 25,000 cells were seeded in each insert. Each treatment condition was set in duplicate for invasion and migration. The lower chamber was replaced with $750 \mu \mathrm{l}$ of complete growth medium $(\alpha-\mathrm{MEM}+5 \%$ FBS $+0.1 \%$ insulin-transferrin-sodium selenite supplement), and the upper chamber was replaced with 25,000 cells resuspended in $500 \mu \mathrm{l}$ medium with no FBS ( $\alpha$-MEM). Following setup, the 24-well plate was incubated for $48 \mathrm{~h}$ at $37^{\circ} \mathrm{C}$ in a low-oxygen $\left(5 \% \mathrm{O}_{2}\right)$ tissue culture incubator. Following the $48 \mathrm{~h}$ incubation, the cells were fixed and stained with toluidine blue in $1 \%$ borax for $3 \mathrm{~min}$. The inserts were imaged using an inverted microscope (IX71; Olympus, NY, USA). Images were taken at nine positions that covered almost the entire area of the insert using a 10 objective. The invaded and migrated cells were counted using the cell counter plugin in Image software (NIH, MD, USA).

\section{RNA sequencing library preparation \& sequencing}

The RNA from the cell lines was extracted from the same passage of cells chosen for DNA extraction (for reduced representation bisulfite sequencing [RRBS]) using a PureLink RNA mini kit (12183025; Invitrogen, CA, USA). The total RNA was extracted according to the manufacturer's instructions. RNA purity was determined by measuring absorbance using a NanoDrop ND-1000 spectrophotometer (Thermo Fisher Scientific, MA, USA). RNA samples with an A260/A280 between 1.8 and 2.0 and A260/A230 close to 2.0 were chosen for downstream analysis. RNA concentration was determined using a Qubit RNA high-sensitivity assay kit (Q32852; Invitrogen) according to the manufacturer's instructions. RNA integrity was determined using a 2100 Bioanalyzer system and associated RNA 6000 Nano kit (5067-1511; Agilent, CA, USA). The RNA integrity numbers of all samples used for the RNA sequencing (RNA-seq) analysis were $\geq 8.0$. The library preparation was done by enrichment of poly-A tailed transcripts from the total RNA, and the library type was strand-specific. A TrueSeq mRNA stranded library prep kit (RS-122-2101; Illumina, CA, USA) was used for mRNA library preparation. Paired-end sequencing of 125 bp was carried out using an Illumina HiSeq 2500 V4 sequencer.

\section{Alignment of RNA-seq data}

Quality of the raw reads was assessed using the FastQC tool. Trimmomatic [31] was used to discard low-quality reads, trim adapter sequences and eliminate poor-quality bases. Tophat2 [32] was used for alignment of RNA-seq data. A prebuilt human genome GRCh37 Bowtie2 index from Illumina's iGenomes collection was used as a reference genome. Gencode 19 annotations were used to annotate all RNAs. 


\section{Differential expression analysis}

The read counts were generated using the htseq-count script, which is a part of the HTSeq Python library [33]. After generating the counts for all the aligned files, the DESeq2 R package was used for the differential expression analysis according to the standard protocol [34]. IncRNAs were annotated using the Gencode 19 annotation file [35]. Fragments per kb million were generated using the Cufflinks pipeline [36].

\section{Protein-protein interaction network}

The STRING database was used for protein-protein interactions. An extended string network was created with 557 differentially expressed genes (DEGs). Minimum interaction score was set to 0.900 . Increased interactors was set to 200. This network was created based on the experimental evidence and database annotations. The number of interactions associated with each node represented the degree of a node. The nodes or proteins with a high degree are considered to be the biological hubs of the network.

\section{Gene Ontology \& pathway analysis}

All differentially expressed protein-coding genes were selected for Gene Ontology (GO) and pathway analysis. This analysis was carried out using the gene list analysis tool in the protein analysis through evolutionary relationships, or PANTHER, database [37]. Fisher's exact test with 5\% false discovery rate (FDR) was applied to retrieve the enriched GO terms and pathways.

\section{Co-expression analysis}

To determine the potential functions of the lncRNAs, a protein-coding gene-lncRNA co-expression network was constructed. The authors calculated Pearson's correlation coefficient between differentially expressed proteincoding genes and lncRNA pairs in R. Pairs with a statistically significant correlation $(\mathrm{p}<0.05)$ were selected for construction of a correlation network. The co-expression network was created using Cytoscape 3.7.1 software. Hub genes were determined using the Matthews correlation coefficient method in the CytoHubba application in Cytoscape 3.7.1.

\section{Cis-target prediction}

The lncRNA-coding gene pairs were defined as cis pairs if the lncRNA-coding gene pair was significantly correlated and the coding genes were within $300 \mathrm{~kb}$ upstream or downstream of any lncRNA.

\section{RRBS library preparation \& sequencing}

Genomic DNA from melanoma cell lines was extracted using a QiAamp DNA mini kit (51104; Qiagen, Hilden, Germany). The 'protocol for cultured cells' was followed according to the manufacturer's instructions with the overnight protein digestion time. The RRBS libraries were prepared using the in-house optimized protocol [38]. Briefly, the extracted genomic DNA was digested with MspI. This step was followed by end repair, A-tailing and adapter ligation. Adapter-ligated MspI fragments were size-selected using the gel-based size selection method after 15-20 cycles, following which they were bisulfite-converted and amplified by PCR. The amplified PCR product was purified and quantified using a Qubit dsDNA high-sensitivity kit (Q32854; Invitrogen). An Agilent Bioanalyzer assay was used to determine the quality of each library and the size distribution of the DNA fragments within each library. Single-ended RRBS libraries with 100 bp read length were sequenced on an Illumina HiSeq 2500 with high output run mode and sequencing by synthesis $\mathrm{v} 4$ chemistry.

\section{Alignment of the RRBS data}

Before alignment, the raw data files were checked run using the FastQC tool (Babraham Institute, Cambridge, UK) to assess the quality of the raw data. Based on the quality reports, adapter and hard trimming was carried out using the cleanadaptors program from the differential methylation analysis package [39]. The alignment was performed using Bismark v0.14.3 [40] against the GRCh37.65 build of the human genome.

\section{Differential methylation analysis}

In this study, the differential methylation analysis package [39] was used for differential methylation analysis of RRBS data. In this approach, 40-220 bp fragment lengths are used as the unit of analysis. The differential methylation analysis package contains two main programs: diffmeth and identgeneloc. To identify differentially methylated 
fragments (DMFs) between the noninvasive and invasive groups of the melanoma cell lines in this study, an analysis of variance test with 5\% FDR was performed using the diffmeth program. The output of diffmeth was used as an input file to the identgeneloc program to relate the fragments to the nearest protein-coding gene. Feature table information regarding protein-coding genes was obtained from the SeqMonk (Babraham Institute) feature table.

\section{Sequenom validation}

The DNA extracted from all nine melanoma cell lines during the RRBS library preparation was saved for the Sequenom validation. Briefly, DNA was extracted from low passage number melanoma cell lines using the QIAamp DNA mini kit (51306; Qiagen). For the eight candidate DMFs, seven primer sets were designed for Sequenom analysis. The primers were designed for bisulfite-converted DNA using the EpiDesigner tool. Bisulfite conversion of genomic DNA was carried out using the EZ DNA methylation kit (D5002; Zymo Research, CA, USA), with the 'alternative cycling protocol' chosen for bisulfite conversion. The bisulfite-converted DNA was amplified by PCR. Before setting up the final PCR conditions for each gene, a temperature gradient PCR optimization was carried out for each gene using a few bisulfite-converted samples. The annealing temperature that provided the maximum amount of product and least primer dimer was selected for the final PCR. The HotStarTaq DNA polymerase kit (203205; Qiagen) was used for PCR. Sequenom assays were carried out by following the EpiTYPER application protocols.

\section{Quantitative real-time PCR validation}

For cDNA synthesis, $1 \mu \mathrm{g}$ of total RNA from each sample was used for generating cDNA in a $20 \mu \mathrm{l}$ reaction. A high-capacity cDNA reverse transcription kit (4368814; Applied Biosystems, CA, USA) was used for synthesizing the first-strand cDNA. The reaction was prepared according to the manufacturer's instructions. SYBR ${ }^{\circledR}$ Premix Ex Taq $^{\text {TM }}$ [Tli RNase H Plus] (RR420W; Takara Bio USA, CA, USA) was used for quantitative real-time PCR (qRT-PCR) according to the manufacturer's instructions. The gene-specific primer concentrations were optimized using three different concentrations of $0.05,0.1$ and $0.2 \mu \mathrm{m}$, following which the $0.2-\mu \mathrm{m}$ final concentration was chosen for both forward and reverse primers. The cDNA samples were diluted 1:4 using DEPC-treated water. In a 96-well quantitative PCR plate (Roche Life Science, Basel, CH), $18 \mu \mathrm{l}$ of SYBR green master mix (Applied Biosystems) was pipetted in triplicate for each gene, followed by the addition of $2 \mu \mathrm{l}$ of cDNA in triplicate. After setting up the PCR plate, the plate was centrifuged briefly. A LightCycler 480 II (Roche Life Science) was used for real-time PCR.

\section{siRNA transfection}

The siRNAs were transfected using a reverse transfection method. The final concentration of siRNA was achieved for each cell line by treating the cells at 5 and $10 \mathrm{~nm}$ siRNA concentrations for 24, 48 and $72 \mathrm{~h}$ time points. The transfection mix, which contained the optimized final concentration of siRNA, Lipofectamine RNAiMAX (13778150; Invitrogen) and Opti-MEM (31985070; Invitrogen), was prepared according to the RNAiMAX manufacturer's instructions. Briefly, the transfection mix was prepared in a tissue culture hood by adding $500 \mu \mathrm{l}$ of Opti-MEM per well of a six-well plate, $2 \mu \mathrm{l}$ of RNAiMAX reagent per well and siRNA according to the chosen concentration in a $15 \mathrm{ml}$ Falcon tube (352095; Corning) and then incubating for $20 \mathrm{~min}$ at room temperature. During the incubation of the transfection mix, cells were harvested by trypsinization and counted using a hemocytometer. Next, $500 \mu \mathrm{l}$ of the transfection mix was added to each well of a six-well plate, followed by dropwise addition of $1.5 \mathrm{ml}$ of cell suspension containing 200,000 cells in each well. The plate was tapped equally on each side to achieve an evenly distributed cell monolayer and then carefully transferred to the low-oxygen culture incubator. To investigate the effect of transfection on cell invasion and migration, an invasion or migration assay was carried out for $48 \mathrm{~h}$ following transfection. The method of the assay was the same as that described earlier.

\section{Integration analysis}

$\mathrm{R}$ was used for the correlation and co-expression analysis. Bedtools closest was used for finding the nearest proteincoding cis-targets of lncRNAs. Bedtools intersect was used to integrate the lncRNAs and the protein-coding, repeat elements and pseudogenes. 

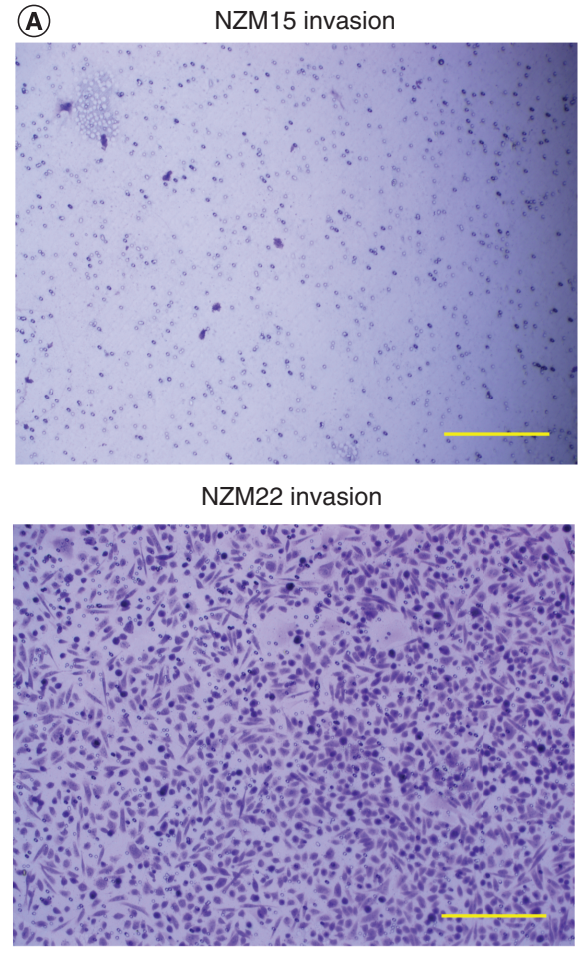

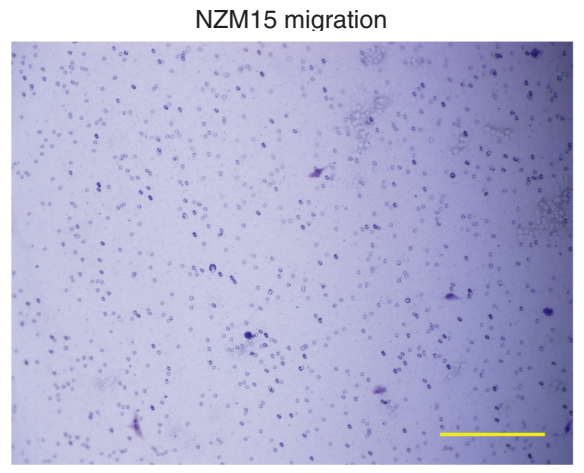

NZM22 migration

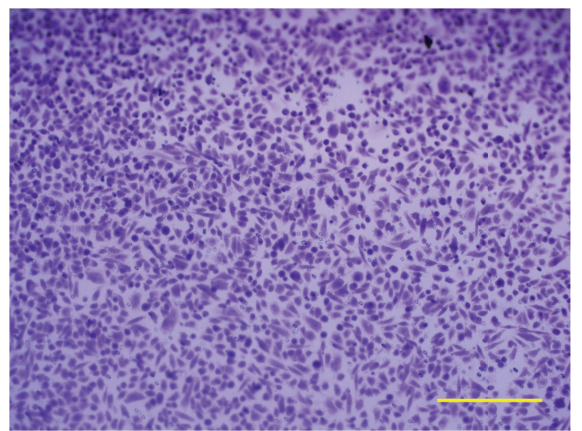

(B)
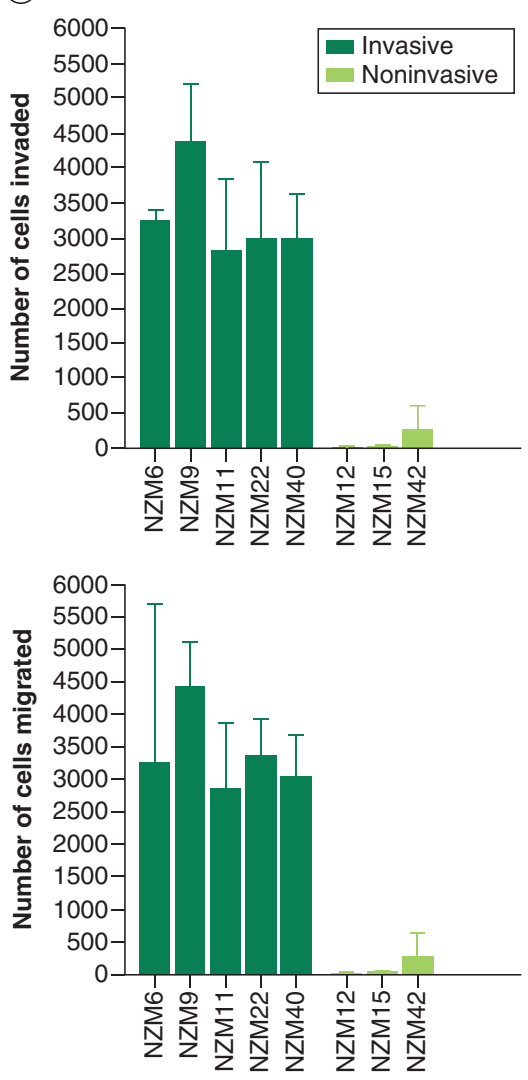

Figure 1. Characterization of noninvasive and invasive phenotypes. (A) Boyden chamber assay images of representative noninvasive and invasive cell lines. The first row shows an image from an invasion assay and a migration assay, respectively, for the noninvasive cell line, NZM15. The second row shows an image from an invasion assay and a migration assay, respectively, for the invasive cell line, NZM22. Scale bar $=500 \mu \mathrm{m}$. (B) Cell invasion and migration assay results of the eight NZM cell lines. The cell invasion/migration assay was performed for $48 \mathrm{~h}$. The error bars represent the standard deviation of the number of cells invading or migrating. The top graph shows the number of cells that had invaded in the invasive and noninvasive groups. The bottom graph shows the number of cells that had migrated in the invasive and noninvasive groups.

Unpaired Mann-Whitney test $\mathrm{p}<0.05$.

NZM: New Zealand melanoma.

\section{Results}

Characterization of invasion \& migration characteristics exhibited by human NZM cell lines

In the first instance, eight melanoma cell lines were analyzed using in vitro Matrigel invasion assays to investigate the relative invasion and migration characteristics. Three cell lines (NZM12, NZM15, NZM42) were found to exhibit relatively low invasion and migratory behaviors (in the range of 30-500 cells that had invaded/migrated in 48 h), whereas five cell lines (NZM6, NZM9, NZM11, NZM22, NZM40) were characterized as exhibiting relatively high invasion and migratory behavior [in the range of 2000-5000 cells that had invaded/migrated in $48 \mathrm{~h}$ ] (Figure 1A \& B). As has been shown previously [6], the classification of the cell lines into groups characterized by either low or high invasive behaviors in vitro did not reflect their mutation status (Supplementary File 1 \& Supplementary Table 1).

Differences in transcription between invasive \& noninvasive human melanoma cell lines identified by RNA-seq analysis

All eight melanoma cell lines were analyzed by RNA-seq. Unsupervised hierarchical clustering of RNA expression profiles was carried out (Figure 2A), which showed that cell lines were grouped by gene expression profiling into multiple clusters, with NZM12, NZM15 and NZM42 in one cluster at the bottom of the dendrogram and NZM6, NZM9, NZM11, NZM22 and NZM40 in groups near the top of the dendrogram. This relative difference in 
(A)
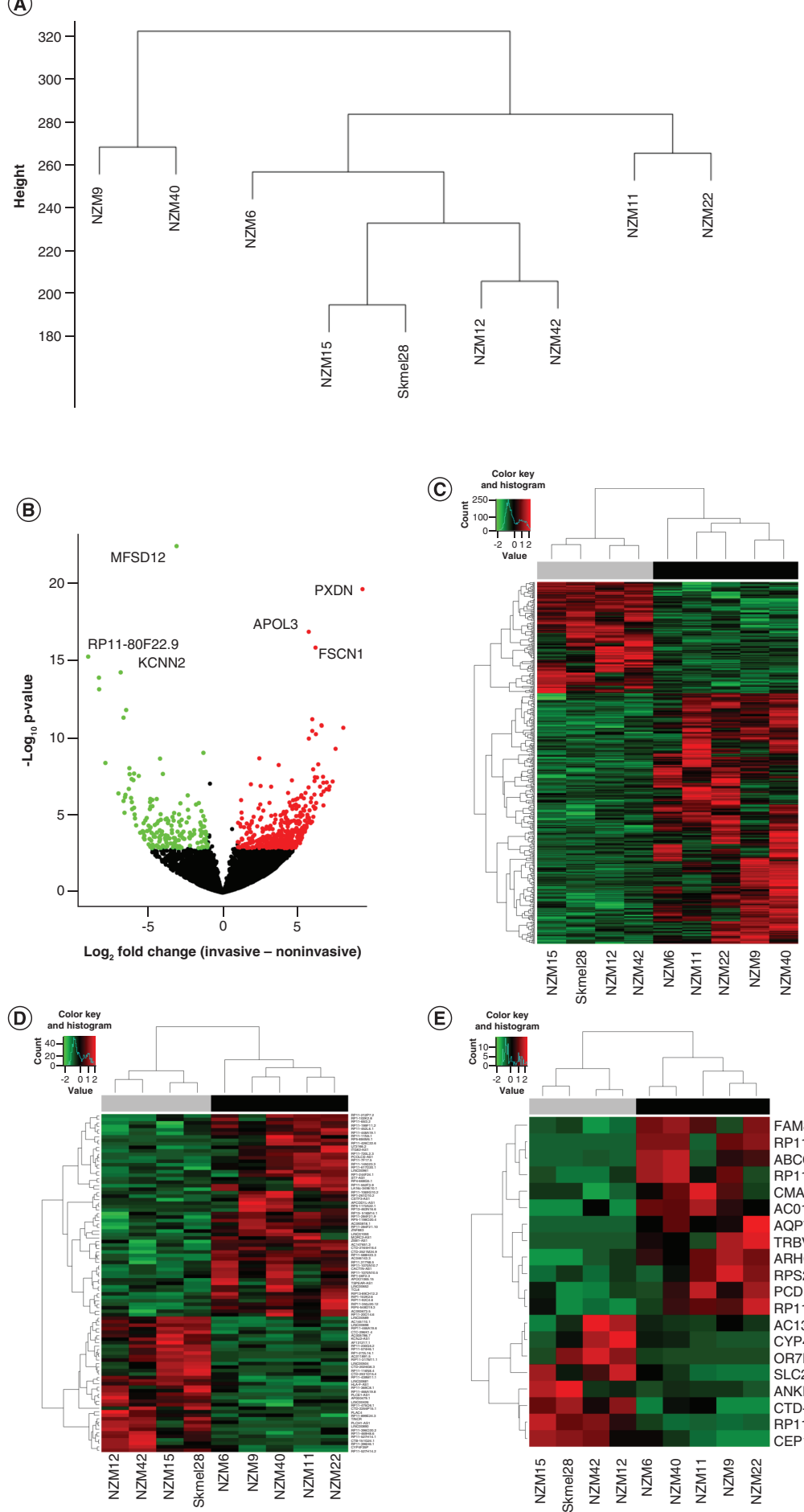

(E)

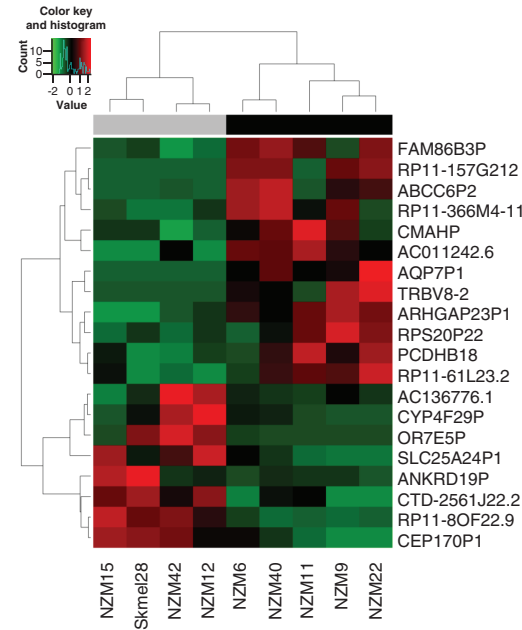

Figure 2. Transcriptome profile of noninvasive and invasive cell lines. (A) Unsupervised clustering (distance metric = Euclidean; linkage method = Complete) of all analyzed RNAs clustered from NZM12, NZM15, NZM42 and SK-MEL-28 in the first group and from NZM9, NZM40, NZM6, NZM11 and NZM12 in the second group. (B) Volcano plot of all analyzed RNAs. X-axis represents log2 of fold change (invasive vs noninvasive), and y-axis represents -log10 of raw p-values. Green dots represent downregulated RNAs, and red dots represent upregulated RNAs. Heatmaps of (C) differentially expressed protein-coding genes, (D) differentially expressed IncRNAs and (E) differentially expressed pseudogenes. The cluster at the top represents the clustering of noninvasive (gray bar) and invasive (black bar) cell lines. Clustering of genes is shown at the left side of the heatmap. The red color represents upregulated RNAs, and the green color represents downregulated RNAs.

NZM: New Zealand melanoma. 
clustering broadly recapitulated the invasion and migration subgrouping of the cell lines. RNA was isolated at the same time from SK-MEL-28 cells, which were grown alongside the other cell lines, and RNA-seq of SK-MEL-28 cells was included with the eight NZM cell lines. Unsupervised hierarchical clustering of the SK-MEL-28 gene expression profile clustered this cell line with the noninvasive melanoma cell lines (Figure 2A). Although phenotypic characterization of the SK-MEL-28 cells for invasiveness was not carried out, subsequent analysis of RNA-seq data from SK-MEL-28 cells using the Heuristic Online Phenotype Prediction database algorithm also classified this cell line as a noninvasive cell line (Supplementary File $1 \&$ Supplementary Table 1).

Differential expression analysis between the nine cell lines was performed (with SK-MEL-28 included in the noninvasive group) using DESeq2 (FDR $\leq 0.05$ and fold change $\geq 2$ ). This identified a total of 557 significantly differentially expressed RNAs across all invasive cell lines compared with noninvasive cell lines (Figure 2B). Of these 557 RNAs, 439 were protein-coding mRNAs, 97 were lncRNAs, 20 were pseudogenes and one was a pri-miRNA (Supplementary File $1 \&$ Supplementary Table 2). Of the 439 differentially expressed protein-coding mRNAs, 304 were upregulated and 135 were downregulated (Figure 2C, Supplementary File $1 \&$ Supplementary Table 3). Additionally, of 97 differentially expressed lncRNAs, 58 were upregulated and 39 were downregulated (Figure 2D). lncRNAs comprised antisense, sense intronic, sense overlap and processed transcripts, with the majority of the lncRNAs being LincRNAs. Of the 20 significantly differentially expressed pseudogenes, 12 were upregulated and eight were downregulated (Figure 2E).

\section{Functional relevance of RNA-seq data}

GO and pathway analysis, including molecular function, biological process and cellular components, and Reactome pathway analysis were carried out to infer functional relevance of the differentially expressed protein-coding mRNAs (Fisher's exact test FDR $\leq 0.05$ ). The top ten GO categories were associated with developmental pathways, transmembrane receptor protein kinase, cell adhesion and extracellular matrix (ECM) features, whereas the top ten pathway categories were associated with ECM organization, collagen formation and immune response. The top ten GO terms and pathways are presented in Supplementary File $1 \&$ Supplementary Table 4.

Further analysis of the functional relevance of the genes was determined through data mining of functional databases. The differentially expressed transcriptome consisted of 11 epigenetic factors, 34 oncogenes, 33 tumor suppressor genes, 22 cancer driver genes, four alternative splicing factors and 19 transcriptional factors (Supplementary File $1 \&$ Supplementary Table 5). The IncRNAs identified in this study were previously reported in manually curated databases that annotated lncRNAs based on experimentally proven functions in important cancer-initiating pathways, such as cell proliferation, cell invasion, apoptosis, metastasis and epithelial-to-mesenchymal transition (EMT), from various cancer types. A total of $23 \mathrm{lncRNAs}$ were previously reported to be associated with melanoma, and many others were reported to be associated with other cancer types and diseases (Supplementary File $2 \&$ Supplementary Table 6).

\section{Protein-protein interaction network \& functional analysis}

To understand how the DEGs might interact with each other in the melanoma cell lines, an extended proteinprotein interaction network was created. In total, 392 nodes and 2191 edges were included in the protein-protein interaction network (Supplementary Figure 1). The genes with the highest degree were PIK3CA (degree $=50$ ), DAP3 $($ degree $=43), M R P L 11$ (degree = 42), PLCG1 $($ degree = 35), C3 $($ degree = 31), CHUK $($ degree $=25), N F K B 1$ $($ degree $=25), P I K 3 C B($ degree $=24), I K B K B($ degree $=24)$ and $I K B K G($ degree $=24)$. The genes were associated with common cancer-related pathways, such as immune response, inflammatory response, MAPK signaling, PI3K signaling, apoptosis, cell migration and viral response. Overlaying immune response genes (Supplementary Figure 1) and ECM genes (Supplementary Figure 1) in the network highlighted most of the genes that were upregulated in the invasive group.

\section{IncRNA co-expression networks}

To explore potential functions of $\operatorname{lncRNAs,~a~co-expression~analysis~was~carried~out~with~the~differentially~expressed~}$ IncRNAs (DELs), differentially expressed pseudogenes and DEGs. Pearson's correlation analysis $(r>0.9$ or $<-0.9$; FDR $=5 \%$ ) identified a total of 1124 lncRNA-mRNA and lncRNA-pseudogene pairs. A co-expression network was then generated using the resultant lncRNA-mRNA and lncRNA-pseudogene pairs, which had 486 nodes and 1124 edges, including all the DELs, 374 unique proteins and 15 unique pseudogenes (Supplementary File $2 \&$ Supplementary Table 7). Genes in the co-expression network were significantly enriched in GO molecular 
functions such as transmembrane receptor protein kinase activity, growth factor binding and ECM constituents; GO biological processes such as cell adhesion and developmental processes and regulation of cell differentiation; and pathways such as cadherin signaling and assembly of collagen fibrils (Supplementary File $2 \&$ Supplementary Table 7). The network analysis revealed 144 nodes of degree 5 and above. The top 20 nodes resulted in a network with 290 nodes and 454 interactions (Supplementary Figure 2). Multiple lncRNAs were correlated with a single gene or pseudogene and vice versa.

\section{Target prediction of cis-regulating IncRNAs}

To investigate the potential cis-regulatory functions of $\operatorname{lncRNAs}$, the nearest protein-coding genes upstream and downstream of lncRNAs were predicted. One of the cis-target networks had 65 nodes and 72 edges (Supplementary File $3 \&$ Supplementary Table 8 \& Supplementary Figure 2) and consisted of 21 protein-coding genes, 43 lncRNAs and one pseudogene. Of the 65 cis-targets, 40 were upregulated and 25 were downregulated. Protein-coding genes in the cis-network were mostly associated with ECM structural proteins, zinc finger transcription factors, ubiquitinprotein ligases, growth factors, protein kinases and actin-binding proteins (Supplementary File 3 \& Supplementary Table 9).

\section{DNA methylation differences between invasive \& noninvasive human melanoma cell lines identified by RRBS global DNA methylation analysis}

Given the relatively large number of gene expression differences and clear phenotypic differences between the noninvasive and invasive cell lines, the authors hypothesized that epigenetic alterations would also be associated with differences between the two groups of cell lines. DNA methylation is the most stable epigenetic modification and marker of cellular identity. The authors carried out genome-wide DNA methylation analysis on the melanoma cell lines (i.e., $\mathrm{n}=4$ noninvasive, including SK-MEL-28, and $\mathrm{n}=5$ invasive cell lines) using RRBS. Greater than 26 million sequence reads were produced for each library, and mapping efficiencies ranged from 62 to $65 \%$. To test the reproducibility of RRBS analysis, two libraries, NZM15 and NZM9, representative of noninvasive and invasive cell line subgroups, respectively, were examined as technical replicates and showed high reproducibility of RRBS (NZM9: Pearson's correlation $=0.95$; NZM15: Pearson's correlation $=0.97$ ). Overall, CpG methylation levels in all RRBS libraries sequenced ranged from 28 to $45 \%$ (Supplementary File $1 \&$ Supplementary Table 10). The RRBS DNA methylation data were also independently validated by choosing specific relevant CpG sites as being representative of a range of DNA methylation values and using Sequenom MassARRAY EpiTYPER. The mean methylation values for CpGs in the fragments that were analyzed by RRBS or Sequenom were highly correlated with each other (Pearson's correlation $=0.86 ; \mathrm{p}<0.0001$ ). Furthermore, as expected, the methylation profiles of the cell lines showed typical bimodal methylation distribution patterns (Supplementary Figure 3).

The overall median methylation level (DNA methylation at all analyzed fragments in the RRBS analysis) in the invasive cell line group was $0.5 \%$ higher than the overall median methylation level in the noninvasive cell line group $\left(\mathrm{p}<2.2 \times 10^{-16}\right)$. Furthermore, median methylation levels in the exon and intron regions were $\sim 8$ and $\sim 2 \%$ higher, respectively, in the invasive cell lines versus the noninvasive cell lines $\left(\mathrm{p}<2.2 \times 10^{-16}\right)$ (Figure 3A). The median methylation levels in the promoter and intergenic regions were $0.2 \%$ higher and $2 \%$ lower, respectively, in the invasive group, but these differences were not statistically significant (Supplementary File $1 \&$ Supplementary Table 11). The global methylation differences were observed in the exon, intron and intergenic regions, and the fact that the majority of the DELs identified were LincRNAs and antisense RNAs prompted the authors to further investigate the overlap of repeat elements with the global methylation profile. All analyzed RRBS fragments $(346,458)$ were further investigated with respect to repeat elements. DNA methylation levels were compared among the various families of these repeat classes. However, the median methylation levels that were observed only in the LTRs associated with the repeat class ERV1 were found to be statistically significantly reduced ( $\sim 7 \%$ lower median methylation in the invasive group; $\mathrm{p}=2.20 \times 10^{-16}$ ) (Figure 3B-E). Of all the fragments overlapping with ERV1, $81 \%$ were in the intergenic regions, and approximately $19 \%$ were in the intron regions, whereas several were in the exon regions. This suggests that the intergenic regions contributed mainly to the hypomethylation of the ERV1 elements in the invasive group.

Unsupervised hierarchical clustering of all analyzed fragments clustered the nine cell lines into different clades, irrespective of the invasive and noninvasive subgroups of the cell lines, suggesting that there was variability of methylation within the subgroups (Supplementary Figure 3). Intergroup differences were further investigated by differential methylation analysis. Analysis of all fragments via ANOVA (CpGs $\geq 2$; reads $\geq 10$; sample count $\geq[\mathrm{n}$ - 
(A)

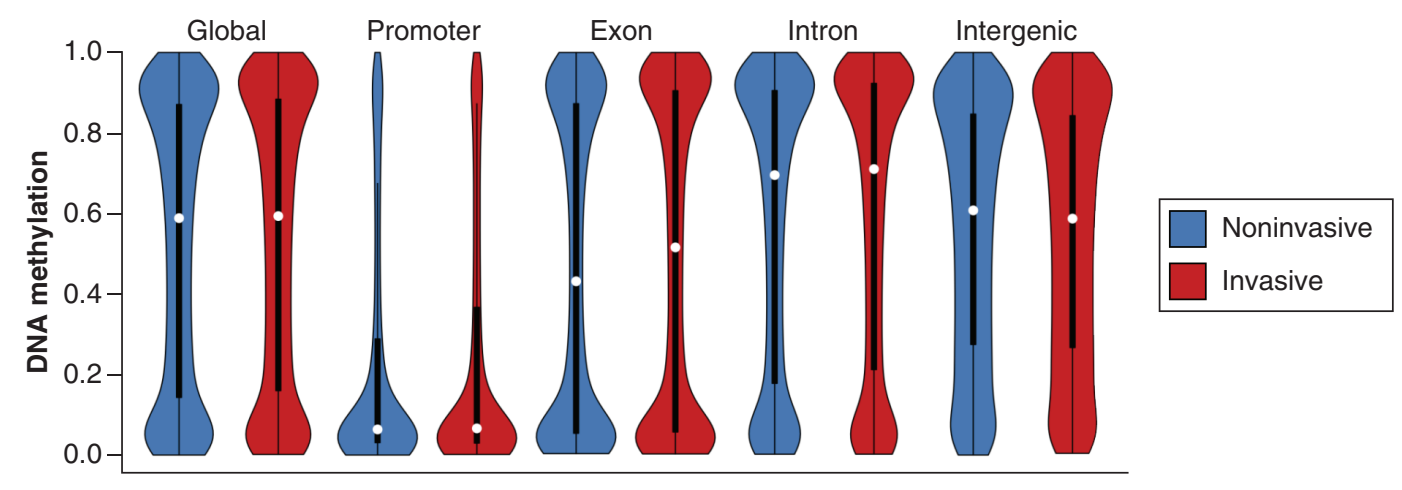

(B)

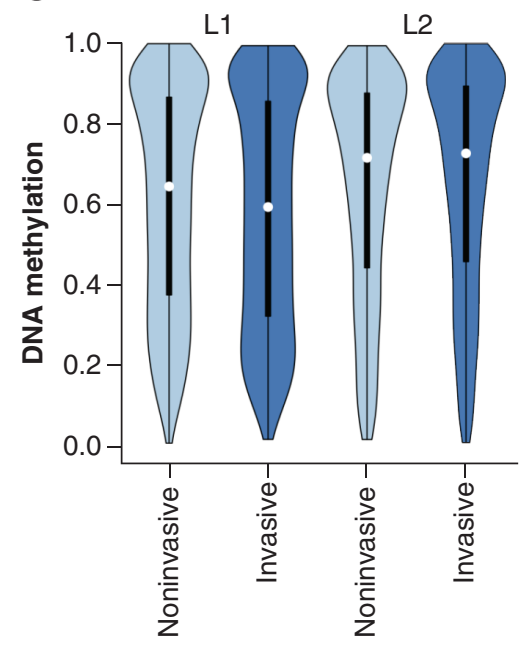

(D)

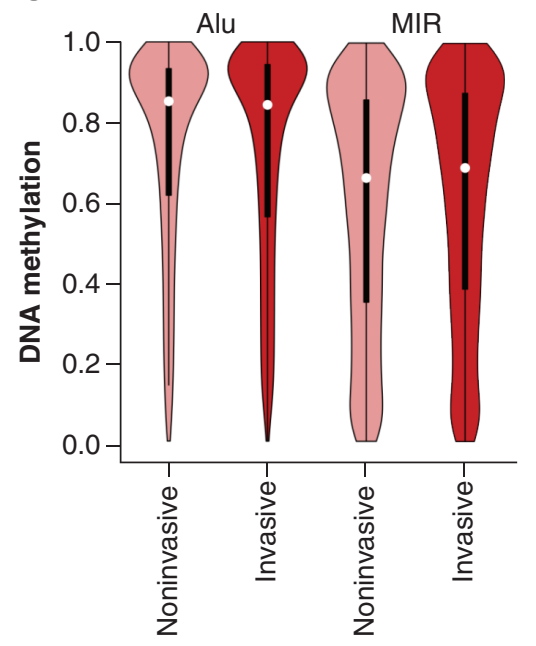

(C)

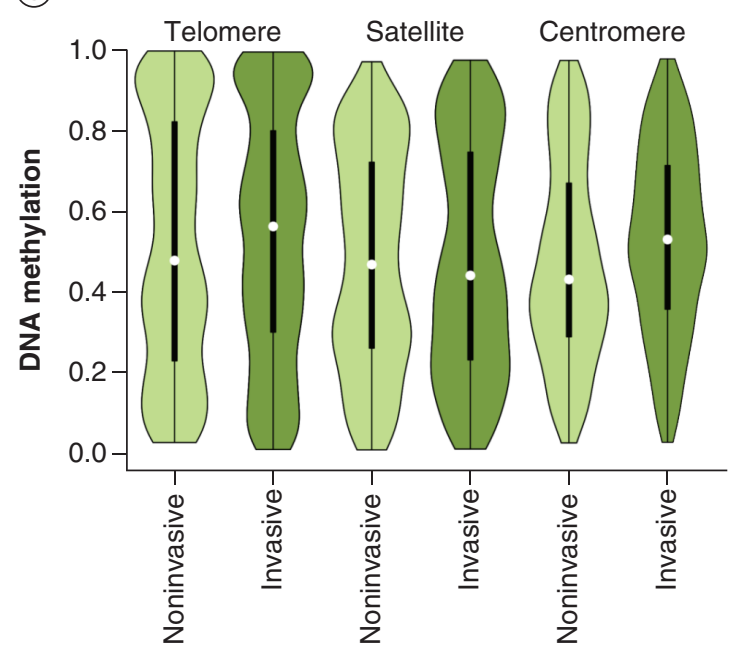

(E)

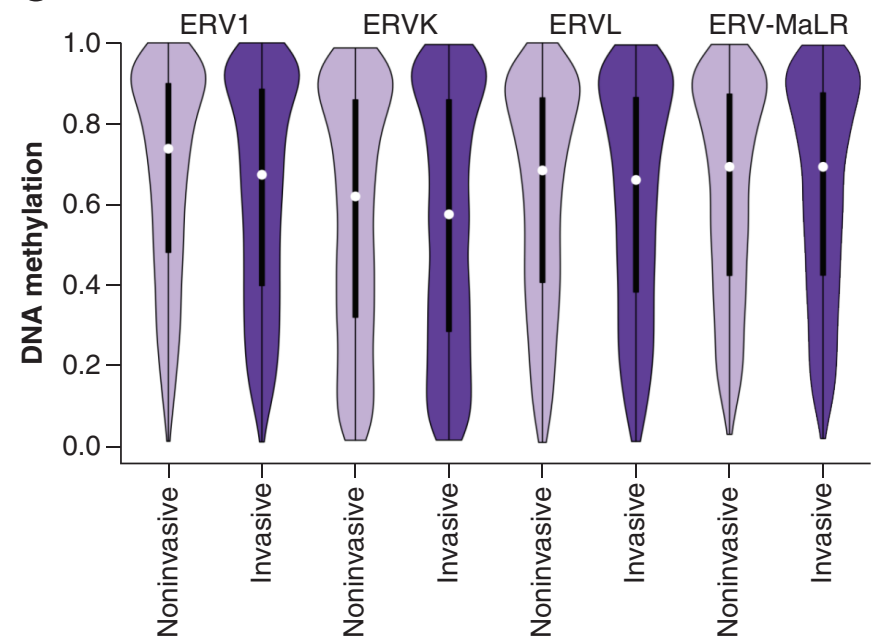

Figure 3. Global methylation profile of invasive and noninvasive cell lines. (A) Violin plots showing distribution of methylation across the genomic element features. Distribution of methylation across the repeat elements, including long interspersed nuclear elements (B), satellite elements (C), short interspersed nuclear elements (D) and long terminal repeats $(E)$, respectively. Y-axis represent the methylation values. The dot in the center of each violin plot represents the median methylation in a group. 

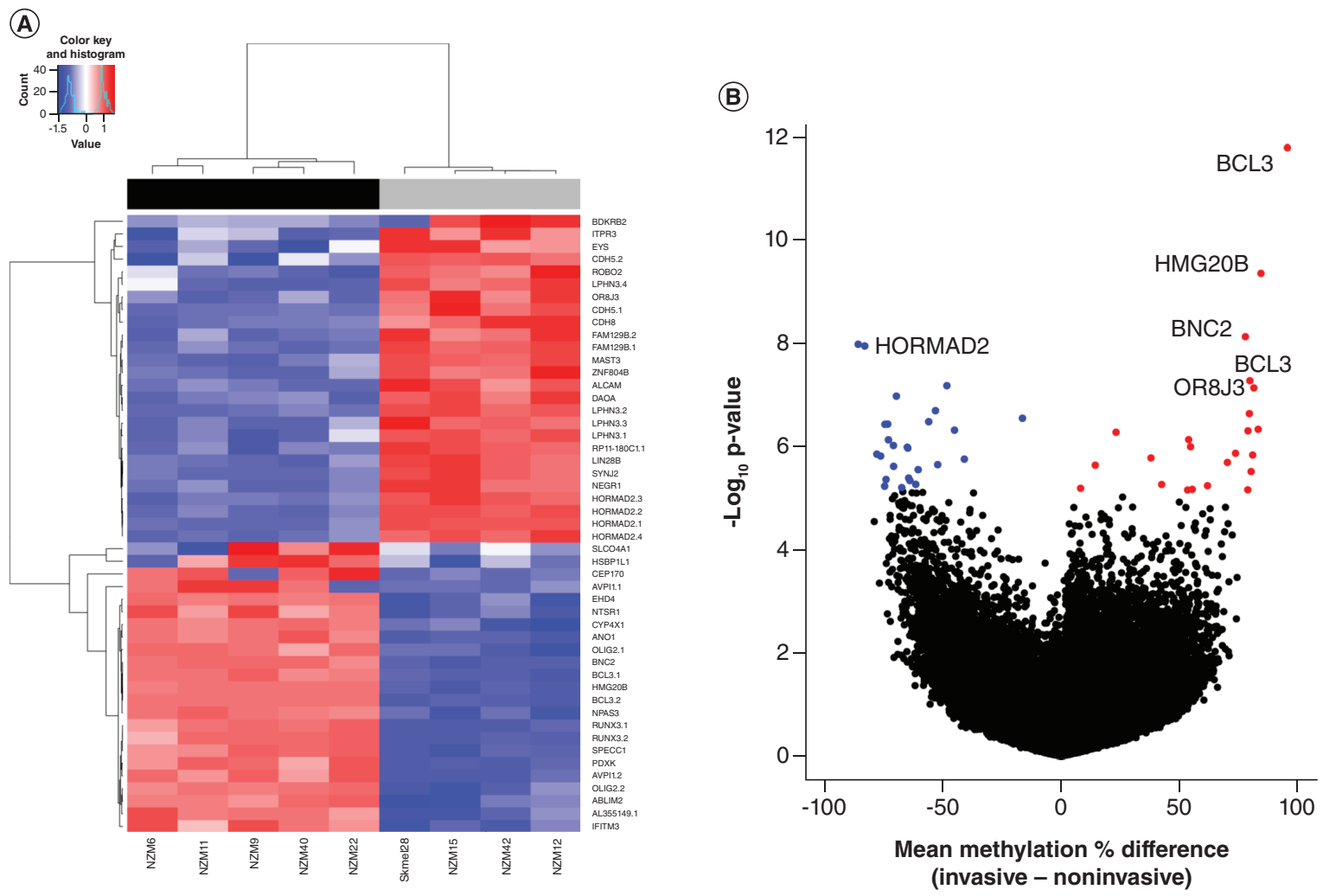

Figure 4. Differential methylation analysis. (A) Heatmap showing the differentially methylated fragments (DMFs). Red represents high methylation and blue represents low methylation. The sample groups are shown by the horizontal bar at the top, with black and gray representing invasive and noninvasive groups, respectively. (B) Volcano plot showing the DMFs in all analyzed fragments. X-axis shows the mean methylation percentage difference between invasive and noninvasive groups. Y-axis shows - $\log 10$ of p-values. All dots in the volcano plot represent all analyzed fragments. The red dots on the right side represent hypermethylated fragments (23 DMFs), whereas the blue dots on the left side represent hypomethylated fragments (26 DMFs).

1]; FDR $=5 \%)$ identified 49 DMFs in invasive cell lines $(n=5)$ compared with noninvasive cell lines $(n=4)$ (Figure 4A, Supplementary File $1 \&$ Supplementary Table 12). In line with the global analysis, $31 \mathrm{DMFs}$ were located in gene bodies (exons and introns), 16 DMFs were located far from transcription start sites (intergenic, defined as $>5 \mathrm{~kb}$ upstream) and two DMFs were within promoter regions (defined as -5 to $+1 \mathrm{~kb}$ ). Of the 49 DMFs, 26 were hypomethylated and 23 were hypermethylated in the invasive group compared with the noninvasive group (Figure 4B). The mean methylation difference in 45 of the DMFs was between 37 and 95\%, whereas the difference in methylation in the remaining four DMFs ranged from 8 to $23 \%$. The DMFs included one epigenetic factor $(H M G 20 B)$ [41], three oncogenes (BCL3, LIN28B, RUNX3) [42], two tumor suppressor genes (CDH5, RUNX3) [43], four cancer driver genes (BCL3, OLIG2, ROBO2, SPECC1) [44], two transcription factor genes (OLIG2, RUNX3) [45,46] and several nondefined DMFs. The top ten DMFs showing the greatest absolute mean methylation difference were BCL3, HORMAD2, HMG20B, AVPI1, RUNX3, CEP170, PDXK, SPECC1, $F A M 129 B$ and $B N C 2$, with mean methylation differences ranging from 0.78 to 0.95 . In the global analysis, several repeat element groups also showed changes in methylation. Upon investigation of the overlap of the DMFs with the repeat elements, of the 49 DMFs, 25 overlapped with repeat elements. Sixteen of these DMFs overlapped with SINEs (Alu = 13; MIRs (mammalian-wide interspersed repeats) = 3), five overlapped with ERVs (ERV1 = 4; ERVL $=1)$ and four overlapped with LINEs $(n=1)$, DNA transposons $(n=1)$, satellites $(n=1)$ and others $(n=1)$.

Integration of methylome \& transcriptome data: mapping of ERV1 DMFs with DEGs \& IncRNAs To identify the effect of ERV1 hypomethylation on expression, the authors mapped the RRBS fragments overlapping with ERV1 to differentially expressed protein-coding genes, lncRNAs and pseudogenes. This was associated with 
an overlap of 106 RRBS fragments occurring in protein-coding regions, which overlapped with 41 differentially expressed protein-coding genes, and 14 RRBS fragments occurring in eight unique differentially expressed lncRNAs. In addition, there were 167 fragments overlapping with ERV1 sequences that were located upstream of 48 DEGs.

Genes identified with both differential expression \& differential methylation

To determine the association between gene expression and DNA methylation, DEGs (439 genes) were compared with DMFs (49 RRBS fragments). Overlaps based on the closest genes to the DMFs revealed six common genes with differential methylation and differential expression (Table 1). The overlapping DMFs were identified in the promoter region (two DMFs) as well as in the intronic region (six DMFs). For example, the mean methylation of two DMFs located in the gene promoter region was inversely correlated with expression of AVPI1 (rho $=-0.67$ and -0.73). In addition, the mean methylation of the six DMFs located in the intronic regions was positively or negatively correlated with expression of their corresponding or closest genes (Spearman's rho: BCL3 $=0.80$, $0.60 ; H M G 2 O B=-0.75 ;$ NTSR1 $=0.62 ;$ ROBO2 $=0.68 ;$ SYNJ2 $=-0.82$ ) (Figure 5). Analysis in the UCSC Genome Browser using ENCODE data showed that these DMFs frequently overlapped with important regulatory regions, such as enhancer regions, transcription factor binding sites, DNA-sensitive regions and histone marks (Supplementary Figure 4). In particular, AVPI1, BCL3, HMG20B, SYNJ2 and NTSR1 overlapped with ENCODE $\mathrm{H} 3 \mathrm{~K} 27 \mathrm{ac}$ and $\mathrm{H} 3 \mathrm{~K} 3 \mathrm{me} 1$ histone marks, transcription factor binding sites (AVPI1- POLR2A; BCL3- SUZ12, ZBTB33; HMG20B- CTCF, CHD4, KDM1A, BCOR, HDAC2, ZMYM3; SYNJ2- ZNF263, GATA2, CTCF, TRIM28, SP1), DNAse I hypersensitivity sites (BCL3, SYNJ2), enhancer regions (AVPI1-GH10J097681, BCL3GH19J044740, HMG20B- GH19J003570, NTSR1- OREG1221880) and CpG islands (BCL3). Furthermore, integration of the individual protein-protein interactions of BCL3, HMG2OB, NTSR1, ROBO2 and SYNJ2, extracted from Supplementary Figure 1, resulted in a single predictive network indicative of how these DMFs may interact with other regulatory proteins.

\section{Validation of expression of selected genes using qRT-PCR}

The expression levels of two genes, AVPI1 and $H M G 20 B$, which were differentially expressed as well as differentially methylated, were validated by qRT-PCR assays. By qRT-PCR, expression of $A V P I 1$ was high in the noninvasive group relative to the invasive group, which confirmed the RNA-seq data for AVPI1. Similarly, HMG2OB was expressed at low levels in the invasive group compared with the noninvasive group in the RNA-seq data, and qRT-PCR validation confirmed these data (Supplementary Figure 5).

\begin{tabular}{|c|c|c|c|c|c|c|c|c|}
\hline Gene name & Location & $\begin{array}{l}\text { Fold change } \\
\text { (log2) (invasive- } \\
\text { noninvasive) }\end{array}$ & $\begin{array}{l}\text { Overlapping differentially } \\
\text { methylated fragments }\end{array}$ & $\begin{array}{l}\text { Genomic } \\
\text { region }\end{array}$ & $\begin{array}{l}\text { Mean } \\
\text { methylation in } \\
\text { Invasive and } \\
\text { noninvasive } \\
\text { groups } \\
\text { respectively }\end{array}$ & $\begin{array}{l}\text { Expression and } \\
\text { methylation with } \\
\text { respect to invasive } \\
\text { group }\end{array}$ & $\begin{array}{l}\text { Correlation } \\
\text { (Spearman' } \\
\text { rank) }\end{array}$ & $\begin{array}{l}\text { Adjusted } \\
\text { p-values }\end{array}$ \\
\hline \multirow[t]{2}{*}{ AVP11 } & \multirow{2}{*}{$\begin{array}{l}\text { chr10:99437181- } \\
99447080\end{array}$} & \multirow[t]{2}{*}{-2.20} & chr10: $99449003-99449070$ & \multirow[t]{2}{*}{ Promoter } & $0.87,0.09$ & \multirow{2}{*}{$\begin{array}{l}\text { Downregulated and } \\
\text { hypermethylated }\end{array}$} & -0.73 & 0.03 \\
\hline & & & chr10: 99449071-99449153 & & $0.91,0.8$ & & -0.90 & 0.004 \\
\hline \multirow[t]{2}{*}{$B C L 3$} & \multirow{2}{*}{$\begin{array}{l}\text { chr19:45250962- } \\
45263301\end{array}$} & \multirow[t]{2}{*}{1.95} & chr19: 45260770-45260850 & \multirow[t]{6}{*}{ Intron } & $0.87,0.07$ & \multirow{2}{*}{$\begin{array}{l}\text { Upregulated and } \\
\text { Hypermethylated }\end{array}$} & 0.80 & 0.01 \\
\hline & & & chr19: 45261562-45261676 & & $0.99,0.03$ & & 0.60 & 0.01 \\
\hline$H M G 2 O B$ & $\begin{array}{l}\text { chr19: } 3572775- \\
3579086\end{array}$ & -2.27 & chr19: 3575342-3575398 & & $0.94,0.10$ & $\begin{array}{l}\text { Downregulated and } \\
\text { Hypermethylated }\end{array}$ & -0.75 & 0.03 \\
\hline NTSR1 & $\begin{array}{l}\text { chr20: } \\
61340189- \\
61394123\end{array}$ & 5.07 & chr20: 61359114-61359247 & & $0.86,0.33$ & $\begin{array}{l}\text { Upregulated and } \\
\text { Hypermethylated }\end{array}$ & 0.62 & 0.09 \\
\hline $\mathrm{ROBO2}$ & $\begin{array}{l}\text { chr3: } 75955846- \\
77699115\end{array}$ & -6.09 & chr3: 77384321-77384413 & & $0.21,0.66$ & $\begin{array}{l}\text { Downregulated and } \\
\text { Hypomethylated }\end{array}$ & 0.68 & 0.05 \\
\hline SYNJ2 & $\begin{array}{l}\text { chr6: } \\
158402888- \\
158520208\end{array}$ & 2.46 & $\begin{array}{l}\text { chr6: } \\
\text { 158447877-158447921 }\end{array}$ & & $0.13,0.86$ & $\begin{array}{l}\text { Upregulated and } \\
\text { Hypomethylated }\end{array}$ & -0.82 & 0.01 \\
\hline
\end{tabular}



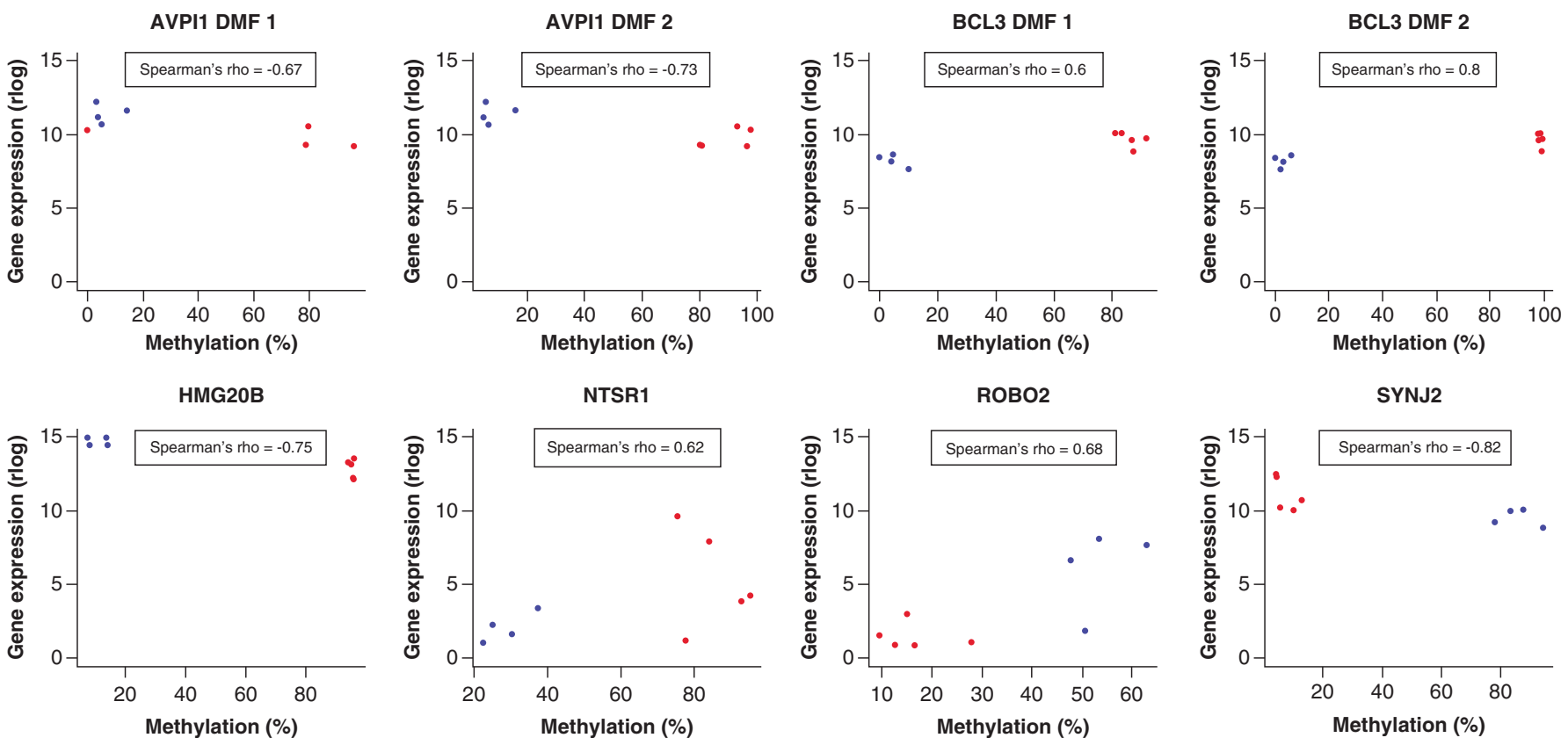

Figure 5. Correlation between differentially expressed genes and differentially methylated fragments. Scatter plots show the correlation of methylation and gene expression across all nine samples using Spearman's rank correlation method.

DMFs: Differentially methylated fragments.

\section{Table 2. Differentially methylated cis-targets.}

\begin{tabular}{|llllcl|}
\hline IncRNA & cis-target gene & $\begin{array}{l}\text { IncRNA fold change } \\
\text { (invasive/noninvasive) }\end{array}$ & $\begin{array}{c}\text { Mean methylation of cis-target } \\
\text { noninvasive and invasive }\end{array}$ & Spearman's rho \\
\hline CACTIN-AS1 & HMG2OB & 4.0 & 0.09 & 0.94 & 0.77 \\
\hline AC005786.7 & HMG2OB & -2.1 & 0.82 & 0.08 & -0.92 \\
\hline RP1-102K2.6 & HORMAD2 & 3.9 & & -0.78 \\
\hline RP1-102K2.8 & HORMAD2 & 3.7 & & -0.57 \\
\hline
\end{tabular}

siRNA-mediated knockdown of AVPI1, investigation of invasion \& migration in noninvasive versus invasive cell lines

An siRNA-mediated knockdown of the AVPI1 gene, confirmed by qRT-PCR (Supplementary Figure 5), resulted in increased cell migration (but not invasion) in a noninvasive cell line, NZM12 ( $<<0.01)$, although the migration difference in NZM15 was not statistically significant. By contrast, NZM42, another noninvasive cell line, although slightly more invasive than NZM12 or NZM15, showed a significant decrease $(\mathrm{p}<0.01)$ in cell migration after knockdown of AVPI1 (Figure 6).

Cis-regulatory IncRNAs \& target genes exhibiting differential methylation

Of the 65 lncRNA cis-target genes, two genes were in common with five DMFs, which were identified in the intronic regions of HMG2OB (one DMF) and HORMAD2 (four DMFs). HMG2OB is a cis-target of antisense lncRNAs, CACTIN-AS1 and AC005786.7, and HORMAD2 is a cis-target of antisense lncRNAs, RP1-102K2.6 and RP1-102K2.8. CACTIN-AS1 and AC005786.7 expression was found to be positively and negatively correlated, respectively, with intronic methylation in $H M G 20 B$ (Spearman's rho $=0.77$ and -0.92 , respectively). By contrast, expression of RP1-102K2.6 and RP1-102K2.8 was negatively correlated with intronic methylation in HORMAD2 (Spearman's rho $=-0.78$ and -0.57 , respectively) (Table 2 ).

\section{Discussion}

Metastatic melanomas are difficult to treat because of their heterogeneous nature. It has previously been shown that melanoma cells present stem-like characteristics, in association with phenotypic heterogeneity, both in vitro and in vivo [47]. Melanomas express genes expressed during embryonic development [48] and can differentiate into 
(A)
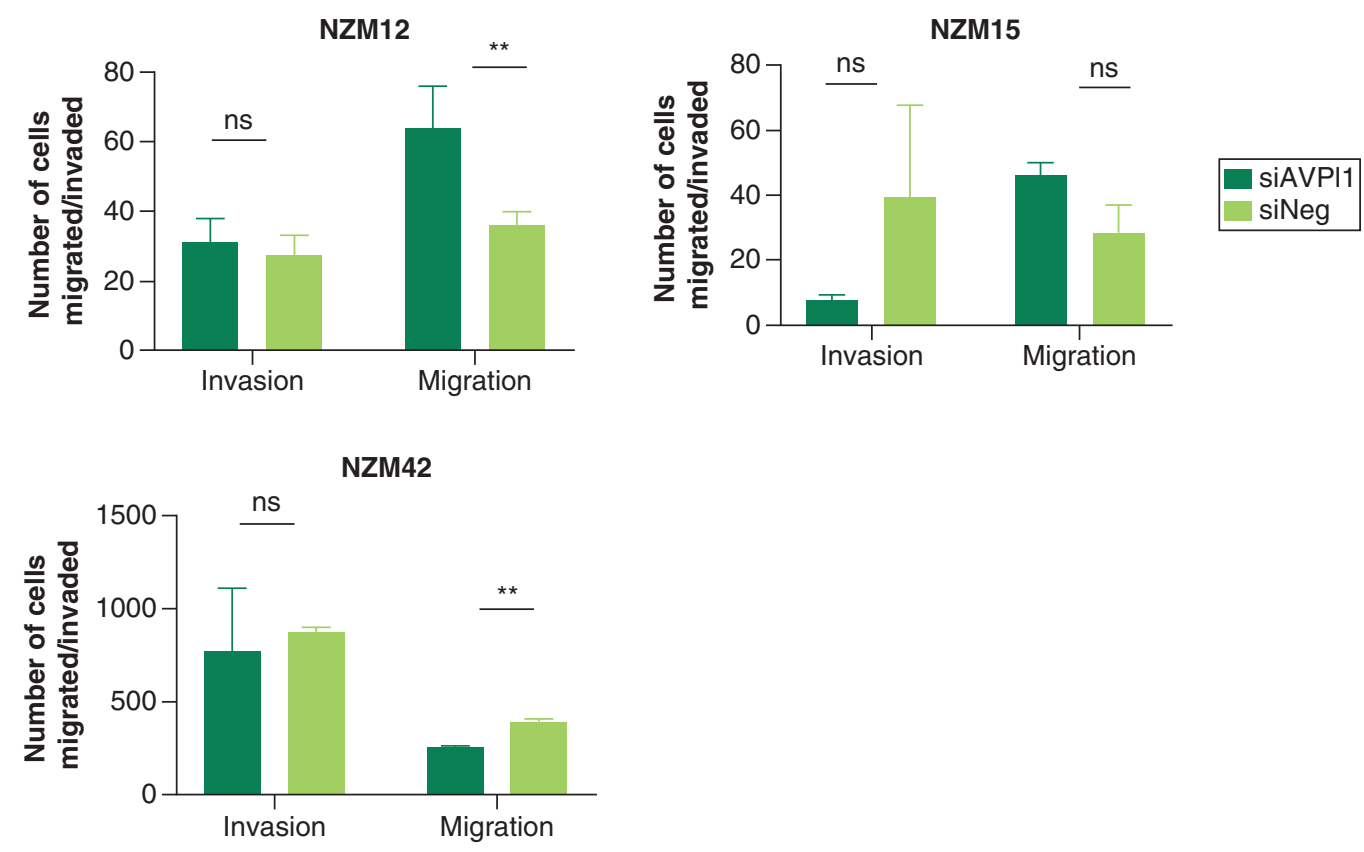

(B)
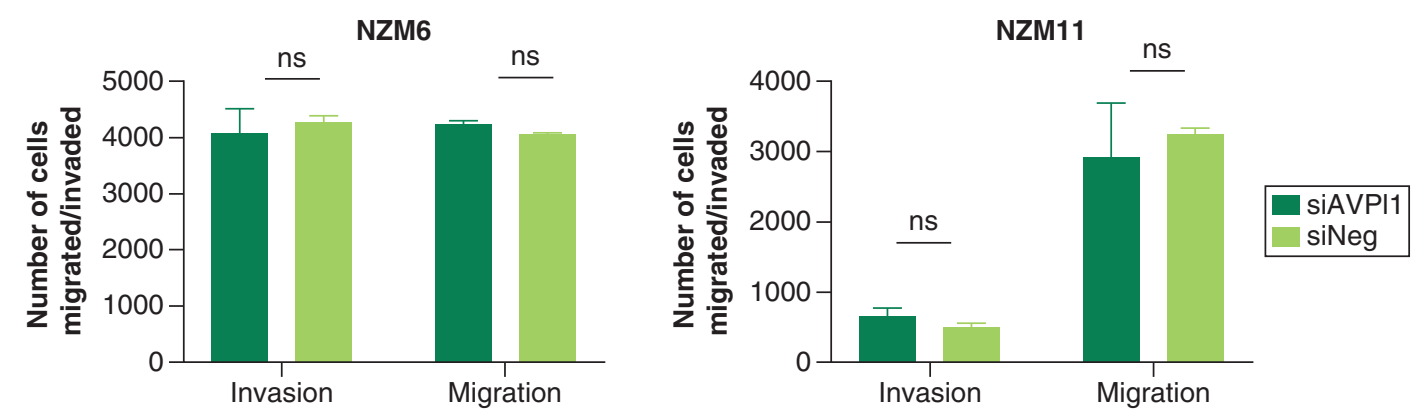

NZM22
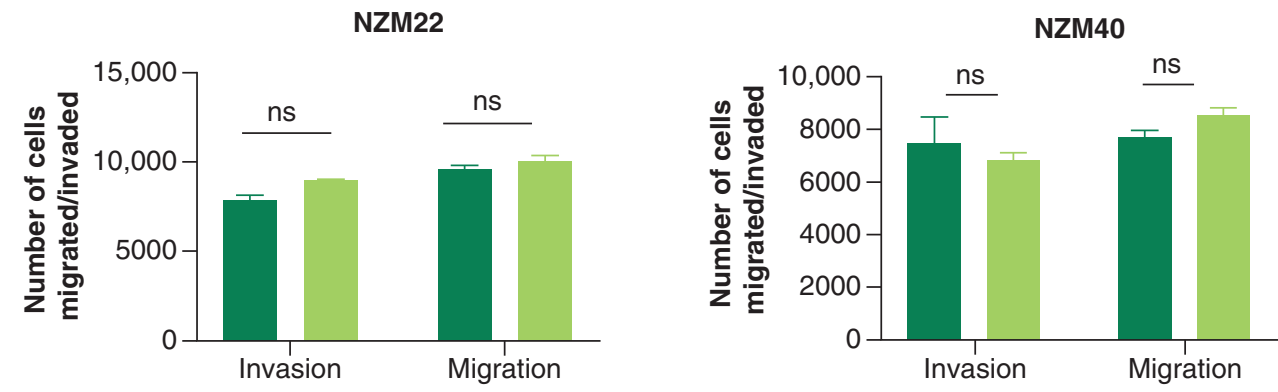

Figure 6. Cell invasion and migration in the noninvasive and invasive cell lines following siRNA knockdown of AVPI1. (A) Cell invasion and cell migration in noninvasive cell lines. (B) Cell invasion and cell migration in invasive cell lines. Y-axis represents the total number of cells that have migrated or invaded. Dark green bars represent the number of cells that have migrated or invaded following AVPI1 knockdown. Light green bars represent the number of cells that have migrated or invaded following treatment with siNeg. Error bars = standard deviation. A paired t-test was applied for statistical significance.

$* * \mathrm{p} \leq 0.01$.

ns: Not significant; NZM: New Zealand melanoma; siNeg: siRNA negative control. 
multiple lineages, including mesenchymal, endothelial and neural [49-51]. However, further investigations carried out on melanoma and other cancers have demonstrated plasticity between nontumorigenic and tumorigenic cancer cells, suggesting that the phenotypic changes are bidirectional in nature, as compared with the unidirectional nature of the cancer stem cell model, supporting a phenotype switching model [30].

Invasiveness is one of the characteristics of metastatic melanomas, but the mechanisms by which melanomas become invasive remain unclear. Recent studies have revealed that in addition to protein-coding genes, the noncoding genome is emerging as a regulatory player in tumorigenesis. This has implications for better understanding the causes underpinning melanoma progression and improving current therapies. In this study, the authors carried out genome-wide transcriptome and methylome analysis of two groups of melanoma cell lines, characterized as noninvasive and invasive, respectively, based on their invasive potential. This integrative study revealed many coding and noncoding genes associated with changes in DNA methylation between the noninvasive and invasive melanoma groups.

Differential expression analysis identified a number of protein-coding genes that are potentially important for cell invasion. Of all the DEGs identified in this study, $54 \%$ were shared by genes identified in previous studies as being associated with invasive and noninvasive phenotypes $[6,28,52]$. Twelve of the genes from the RNA-seq data matched a multicancer invasion, 64-gene signature that is associated with common epithelial cancers, including ovarian, colon, breast, gastric and lung cancer, and some nonepithelial cancers, such as neuroblastoma and Ewing's sarcoma (Supplementary File $1 \&$ Supplementary Table 13). Moreover, these genes were among the top 100 upregulated and top 100 downregulated DEGs in this study [53,54]. DEGs also included EMT transcription factors such as $Z E B 1$ and $S N A I 1$, as described in previous studies [55,56]. Strikingly, PXDN, which is a fibroblast-associated protein involved in the formation of ECM [57], had the highest relative expression in the invasive group. Recent studies suggest that PXDN has a crucial role in melanoma invasion in vitro and in vivo [58]. In addition, DEGs included several hypoxia signature genes previously reported in breast cancer [59].

Differentially expressed RNAs also included noncoding genes such as DELs and differentially expressed pseudogenes. The majority of the DELs were LincRNAs, in which transposable elements are frequently active as regulatory regions [27], and antisense lncRNAs. Antisense lncRNAs occur frequently in about $60-70 \%$ of the transcribed regions of the human genome. Moreover, antisense transcripts have been shown to epigenetically regulate gene expression, and alterations in their expression can lead to the silencing of important regulatory genes, such as tumor suppressor genes [60,61]. Antisense lncRNAs have also been observed to be derived from pseudogenes [62,63], and some studies have reported copy number variations and gene expression alterations of pseudogenes in cancer [64]. Although there may potentially be only marginal functional relevance of the 20 pseudogenes identified in this study, eight have previously been associated with melanoma (AQP7P1 [65], FAM86B3P [64], OR7E5P [66], PCDHB18 [67]), hypoxia (RP11-61L23.2 [59]), prostate cancer (RP11-366M4.11 [68]), antiviral mechanisms (RP11-157G21.2 [69]) and Huntington's disease (RPS20P22 [70]).

Co-expression and target prediction analysis revealed a number of interesting targets and hub proteins as potentially key regulators of biological networks. Among lncRNAs identified in the networks was the oncogene TINCR [71], the EMT regulators ZEB1-AS1 [72] and ITGB2-AS1 [73] and the tumor suppressor gene TCL6 [74]. TINCR [75] and LINC00589 [76] have been associated with major hallmark pathways of cancer, such as cell proliferation, apoptosis, cell migration, cell invasion and EMT. LINC00961 is associated with positive regulation of patient survival in renal carcinoma and negative regulation of cancer progression and EMT [77-79]. LINC00681 was identified as the top hub lncRNA, with 31 interactions, and it has previously been associated with epithelial cancer and neurological disorders (Supplementary Table 6). In a recent study, LINC00681, along with several other IncRNAs, was shown to be differentially expressed in uveal melanoma subtypes [80]. The authors also found that LINC00681 was co-expressed with AVPI1 and MFSD12, which is located upstream of HMG20B. In this regard, there were two different DMFs in this study associated with $A V P I 1$ and $H M G 20 B$. Additionally, 29 other lncRNAs were co-expressed with DMFs (HMG20B, SYNJ2, BCL3, ROBO2, NTSR1 and AVPI1) or genes upstream or downstream of DMFs (BCAM, MFSD12, CACTIN-AS1, CTD-2026G6.3 and AC005786.7) (Supplementary File $2 \&$ Supplementary Table 7). PCOLCE-AS1, which interacts with BCL3, is among the top five hub proteins and is known to be a super-enhancer in ovarian cancer [81]. Co-expression analysis also identified six lncRNAs (LINC00698, ST7-AS1, CSTF3-AS1, LINC00504, KCNJ2-AS1 and PLCH1-AS1) that were previously identified as cancer- or lineage-associated lncRNAs in a large cohort of cancer patient datasets from The Cancer Genome Atlas, ENCODE and Gene Expression Omnibus databases [82]. 
The majority of the identified cis-regulating lncRNAs exhibited a high degree of interactions (Supplementary File $3 \&$ Supplementary Table 8). Of the 43 cis-regulating lncRNAs identified, 12 were previously reported in melanoma, and 31 have previously been reported in other cancers (Supplementary File $2 \&$ Supplementary Table 6). The cis-regulated targets included epigenetic factors, such as $H M G 2 O B$ [41] and $U B E 2 H$ [83]; tumor suppressor genes, such as GOS2 [84]; cancer driver EMT genes and transcription factors, such as $Z E B 1$ [85]; pseudogenes associated with hypoxia, such as RP11-61L23.2 [59]; ECM organization genes involved in cancer, such as COL15A1, COLEC12 and PCOLCE; growth factor binding proteins, such as FAM20C; genes involved in oncogenic signaling of the MAPK pathway, such as FAM131B; and genes involved in interferon signaling, such as NUB1 and HLA-G (Supplementary File $3 \&$ Supplementary Table 9). NUB1 encodes an interferon-inducing protein, which is known to inhibit proliferation in renal cell carcinoma [86].

Global methylation profiling identified ERV1 retrotransposon hypomethylation in the invasive cell line group. ERV1-containing retrotransposons belong to a class of expressed repeat element-containing loci that frequently overlap with differentially expressed protein-coding genes and lncRNAs. In addition, some ERV1-containing fragments can be found upstream of protein-coding genes. Insertions of ERVs in protein-coding genes and IncRNAs can lead to alternative splicing and alternative promoter activity [87]. Furthermore, ERVs in upstream regions of genes can act as enhancers [88].

Most of the DMFs that overlapped with DEGs (AVPI1, HMG2OB, SYNJ2, BCL3) appeared likely to be crucial in gene regulatory functions, as they overlapped with transcription factor binding sites, enhancer regions, histone marks and DNAse I hypersensitivity sites. SYNJ2 is a member of the 5 -inositol lipid phosphatase family, which has been reported to promote cell migration and invasion in breast cancer and is associated with a high risk of colorectal cancer [89,90]. Interestingly, the DMFs associated with SYNJ2 and HMG2OB overlap with CTCF transcription factor binding sites. CTCF is known to affect transcriptional outcomes and to regulate chromatin structure [91]. $B C L 3$ functions as a transcriptional co-activator, and its high expression is correlated with poor prognosis in colorectal cancer and gliomas [92,93]. The DMFs associated with BCL3 overlap with ZBTB33, which has been reported to be a reader of methylated DNA [94] and to have a role in cancer [95]. NTSR1 belongs to the family of G-coupled receptors, and hypermethylation of NTSR1 has been associated with noninvasiveness in colorectal cancers [96]. $\mathrm{ROBO} 2$ is a transmembrane receptor and is associated with axon guidance and cell migration. $\mathrm{ROBO} 2$ is hypomethylated in gastric cancer tissue compared with pericancerous tissue [97]. In the authors' investigation involving invasive melanoma cell lines, the DMF located in an NTSR1 intron was hypermethylated, whereas the $\mathrm{DMF}$ in an intron in $\mathrm{ROBO} 2$ was hypomethylated, and $\mathrm{ROBO} 2$ expression was reduced in invasive melanoma cell lines compared with noninvasive melanoma cell lines (Supplementary Table 12).

Interestingly, one of the DMFs was identified in an intron region of $H M G 20 B$, which has been reported to be an epigenetic factor involved in chromatin organization [41] and is required for cell division and cytokinesis, in association with BRCA2 [98]. Hypomethylation of $H M G 20 B$ was positively correlated with gene expression. This DMF region overlaps with regions involved in epigenetic regulation, including HDAC2, an epigenetic repressor that is involved in regulating transcription and cell cycle progression [99]. It also overlaps with the epigenetic modifier KDM1A, which is essential for cell growth and interacts with promoter and enhancer regions [100], and CHD4, which is known to be involved in epigenetic repression. Mutations in $C H D 4$ are associated with tumorigenesis [101].

Target prediction analysis of IncRNAs identified CACTIN-AS1 and ACO05786.7 as cis-regulating IncRNAs of $H M G 20 B$. Furthermore, the co-expression analysis identified three lncRNAs - namely, AP000479.1, LINC00426 and RP11-317M11.1 - which were co-expressed with HMG20B. Of the three lncRNAs, LINC00426 and RP11$317 M 11.1$ were also co-expressed with MFSD12, which is upstream of HMG2OB. MFSD12 is also one of the target genes for the predicted enhancer region (GH19J003570) from the GeneHancer database overlapping the DMF in the $H M G 20 B$ intron region. Recent work has identified MFSD12 as a crucial gene for melanocyte function [102]. MFSD12 is the top gene in the author' dataset showing downregulation in the invasive group. Taken together, the aforementioned evidence suggests that there is a relationship between the expression of the cis-regulating and co-expressed lncRNA targets of $H M G 20 B$, the expression of MFSD12 and the high methylation levels of an intron region in $H M G 20 B$ in the invasive group.

The AVPI1 promoter contains several DMFs that were hypermethylated in the invasive cell line group. AVPII mRNA was also differentially expressed, with low expression in the invasive group. AVPI1 knockdown has been reported to significantly inhibit the induction of cell death by MLN4924, an inhibitor of the NEDD8-activating enzyme, which is involved in cancer progression [103]. Furthermore, high levels of AVPI1 expression have been identified in association with cell cycle entry [104] and as being involved in activating the MAPK pathway [105,106]. 
Knockdown of AVPI1 in invasive melanoma cells had little or no effect on invasiveness, potentially because $A V P I 1$ expression levels were already low. By contrast, AVPI1 knockdown in the noninvasive cell line NZM12 resulted in significantly increased migration. In contradiction to this, AVPI1 knockdown decreased migration in NZM42, which was classified in this study as noninvasive, although this cell line was more invasive than NZM12 or NZM15 and was shown to exhibit a gene expression pattern that was intermediate between the invasive and noninvasive groups and termed 'transitory' [4]. AVPI1 expression was highly correlated with LINC00681 lncRNA, which was identified in the largest hub node in the cis-regulating network, and LINC00681 was co-expressed with 30 other coding/noncoding genes. This lncRNA has also been reported to be significantly co-expressed in uveal melanomas. In addition, expression levels of two other lncRNAs - namely, RP11-396020.2 and PLAC4 - were significantly correlated with AVPI1.

\section{Conclusion}

In this study, NZM cell lines with invasive behavior were found to exhibit differential expression of both coding and lncRNAs compared with noninvasive cell lines. Changes in methylation levels were also observed in invasive versus noninvasive cell lines, particularly with regard to the hypomethylation of some repeat elements (e.g., ERV1) in the intergenic regions and hypermethylation of introns and coding regions of genes. In total, 49 DMFs were identified between the invasive and noninvasive cell lines; of these, 12 were identified as overlapping with DEGs or DELs. One of these genes, AVPI1, was investigated for a functional role in migration or invasion, and some evidence supported a role for AVPI1 in cell migration in one cell line, NZM12. Taken together, these data demonstrate DNA methylation changes and associated changes in the expression of cRNAs as well as ncRNAs, which correlates with invasiveness in melanoma cell lines.

\section{Future perspective}

In this study, we have identified $12 \mathrm{DMFs}$ that are highly correlated with gene expression between noninvasive and invasive phenotypes in melanoma. These candidates may represent potential therapeutic targets in melanoma therapy. Further studies will be required to investigate the mechanism of their involvement in melanoma invasion.

\section{Summary points}

- Transcriptome analysis by RNA sequencing identified 557 transcripts, which included 439 protein-coding genes, 97 IncRNAs, 20 pseudogenes and one pri-miRNA transcript.

- Transcriptome data were enriched in Gene Ontology categories associated with developmental pathways, transmembrane receptor protein kinase, cell adhesion and extracellular matrix features and pathway categories associated with extracellular matrix organization, collagen formation and immune response.

- Targets for cis-regulating IncRNAs were predicted. The cis-target network had 65 nodes and 72 edges. Protein-coding genes in the cis-network were mostly associated with extracellular matrix structural proteins, zinc finger transcription factors, ubiquitin-protein ligases, growth factors, protein kinases and actin-binding proteins.

- Global methylation differences were observed in exon, intron and intergenic regions. ERV1 elements were hypomethylated predominantly in the intergenic regions in the invasive cell lines compared with the noninvasive cell lines.

- Methylome analysis by reduced representation bisulfite sequencing identified 49 differentially methylated fragments. Integrated analysis revealed association of transcriptome data with 12 differentially methylated fragments, represented by high correlation corresponding with or closest to nine genes or IncRNAs.

- The AVPI1 promoter contained several differentially methylated fragments that were hypermethylated in the invasive cell line group. AVPI1 mRNA was also differentially expressed, with low expression in the invasive group.

- AVPI1 knockdown in a noninvasive cell line, NZM12, was associated with increased cell migration, although more investigations are required to determine the role of AVPI1 in cell migration.

Supplementary data

To view the supplementary data that accompany this paper please visit the journal website at: www.futuremedicine.com/doi/suppl/10.2217/epi-2020-0440

Author contributions

Conceptualization: J Motwani, E J Rodger, E C Macaulay, P A Stockwell and M R Eccles. Methodology, investigation, interpretation and validation: J Motwani. Design: J Motwani and M R Eccles. Analysis: J Motwani, E J Rodger and P A Stockwell. Visualization: 
J Motwani and E J Rodger. Writing of original draft: J Motwani. Review and editing: J Motwani, M R Eccles, E C Macaulay, P A Stockwell and B C Baguley. Resources: M R Eccles and B C Baguley. Funding acquisition and supervision: M R Eccles. All authors have read and approved the final manuscript.

\section{Acknowledgments}

The authors thank M Parry for his advice on statistical analysis of methylation data.

\section{Financial \& competing interests disclosure}

This work was supported by the Maurice Wilkins Centre for Molecular Biodiscovery, Lottery Health Research New Zealand, Healthcare Otago Charitable Trust, Maurice and Phyllis Paykel Trust and H S and J C Anderson Trust. The authors have no other relevant affiliations or financial involvement with any organization or entity with a financial interest in or financial conflict with the subject matter or materials discussed in the manuscript apart from those disclosed.

No writing assistance was utilized in the production of this manuscript.

Ethical conduct of research

The authors state that they have obtained appropriate institutional review board approval or have followed the principles outlined in the Declaration of Helsinki for all human or animal experimental investigations. In addition, for investigations involving human subjects, informed consent has been obtained from the participants involved.

\section{Open access}

This work is licensed under the Attribution-NonCommercial-NoDerivatives 4.0 Unported License. To view a copy of this license, visit http://creativecommons.org/licenses/by-nc-nd/4.0/

Data availability statement

RNA sequencing and DNA methylation datasets have been deposited in the National Center for Biotechnology Information Gene Expression Omnibus with accession number GSE153595.

\section{References}

1. Apalla Z, Lallas A, Sotiriou E, Lazaridou E, Ioannides D. Epidemiological trends in skin cancer. Dermatol. Pract. Concept. 7(2), 1-6 (2017).

2. Rousseau DL Jr., Ross MI, Johnson MM et al. Revised American Joint Committee on Cancer staging criteria accurately predict sentinel lymph node positivity in clinically node-negative melanoma patients. Ann. Surg. Oncol. 10(5), 569-574 (2003).

3. Marshall ES, Holdaway KM, Shaw JH, Finlay GJ, Matthews JH, Baguley BC. Anticancer drug sensitivity profiles of new and established melanoma cell lines. Oncol. Res. 5(8), 301-309 (1993).

4. Tran KB, Gimenez G, Tsai P et al. Genomic and signalling pathway characterisation of the NZM panel of melanoma cell lines: a valuable model for studying the impact of genetic diversity in melanoma. Pigment Cell Melanoma Res. 34(1), 136-143 (2021).

5. Tsoi J, Robert L, Paraiso $\mathrm{K}$ et al. Multi-stage differentiation defines melanoma subtypes with differential vulnerability to drug-induced iron-dependent oxidative stress. Cancer Cell 33(5), 890-904.e895 (2018).

6. Jeffs AR, Glover AC, Slobbe LJ et al. A gene expression signature of invasive potential in metastatic melanoma cells. PLoS One 4(12), e8461 (2009)

7. Lee E, Iskow R, Yang L et al. Landscape of somatic retrotransposition in human cancers. Science 337(6097), 967-971 (2012).

8. Lander ES, Linton LM, Birren B et al. Initial sequencing and analysis of the human genome. Nature 409, 860 (2001).

9. Slotkin RK, Martienssen R. Transposable elements and the epigenetic regulation of the genome. Nat. Rev. Genet. 8(4), 272-285 (2007).

10. Esteller M, Herman JG. Cancer as an epigenetic disease: DNA methylation and chromatin alterations in human tumours. J. Pathol. 196(1), 1-7 (2002).

11. Wolff EM, Byun HM, Han HF et al. Hypomethylation of a LINE-1 promoter activates an alternate transcript of the MET oncogene in bladders with cancer. PLoS Genet. 6(4), e1000917 (2010).

12. Roman-Gomez J, Jimenez-Velasco A, Agirre X et al. Promoter hypomethylation of the LINE-1 retrotransposable elements activates sense/antisense transcription and marks the progression of chronic myeloid leukemia. Oncogene 24, 7213 (2005).

13. Lin CH, Hsieh SY, Sheen IS et al. Genome-wide hypomethylation in hepatocellular carcinogenesis. Cancer Res. 61(10), 4238-4243 (2001).

14. Santourlidis S, Florl A, Ackermann R, Wirtz HC, Schulz WA. High frequency of alterations in DNA methylation in adenocarcinoma of the prostate. Prostate 39(3), 166-174 (1999). 
15. Macaulay EC, Roberts HE, Cheng X, Jeffs AR, Baguley BC, Morison IM. Retrotransposon hypomethylation in melanoma and expression of a placenta-specific gene. PLoS One 9(4), e95840 (2014).

16. Lavie L, Kitova M, Maldener E, Meese E, Mayer J. CpG methylation directly regulates transcriptional activity of the human endogenous retrovirus family HERV-K(HML-2). J. Virol. 79(2), 876-883 (2005).

17. Dong J, Sedrak M, Rincon L, Sun H, Garza G, Kelly B. K-type human endogenous retroviral elements in human melanoma. $A d v$. Genomics Genet. 4, 153-159 (2014).

18. Wang-Johanning F, Radvanyi L, Rycaj K et al. Human endogenous retrovirus K triggers an antigen-specific immune response in breast cancer patients. Cancer Res. 68(14), 5869-5877 (2008).

19. Hu L, Hornung D, Kurek R, Östman H, Blomberg J, Bergqvist A. Expression of human endogenous gammaretroviral sequences in endometriosis and ovarian cancer. AIDS Res. Hum. Retrovir. 22(6), 551-557 (2006).

20. Roulois D, Loo yau H, Singhania $\mathrm{R}$ et al. DNA-demethylating agents target colorectal cancer cells by inducing viral mimicry by endogenous transcripts. Cell 162(5), 961-973 (2015).

21. Wang-Johanning F, Frost AR, Jian B et al. Detecting the expression of human endogenous retrovirus E envelope transcripts in human prostate adenocarcinoma. Cancer 98(1), 187-197 (2003).

22. Shukla R, Upton KR, Munoz-Lopez M et al. Endogenous retrotransposition activates oncogenic pathways in hepatocellular carcinoma. Cell 153(1), 101-111 (2013).

23. Panda A, De Cubas AA, Stein M et al. Endogenous retrovirus expression is associated with response to immune checkpoint blockade in clear cell renal cell carcinoma. JCI Insight 3(16), e121522 (2018).

24. Strissel PL, Ruebner M, Thiel F et al. Reactivation of codogenic endogenous retroviral (ERV) envelope genes in human endometrial carcinoma and prestages: emergence of new molecular targets. Oncotarget 3(10), 1204-1219 (2012).

25. Solovyov A, Vabret N, Arora KS et al. Global cancer transcriptome quantifies repeat element polarization between immunotherapy responsive and T cell suppressive classes. Cell Rep. 23(2), 512-521 (2018).

26. Morlando M, Fatica A. Alteration of epigenetic regulation by long noncoding RNAs in cancer. Int. J. Mol. Sci. 19(2), 570 (2018).

27. Hadjiargyrou M, Delihas N. The intertwining of transposable elements and non-coding RNAs. Int. J. Mol. Sci. 14(7), 13307-13328 (2013).

28. Verfaillie A, Imrichova H, Atak ZK et al. Decoding the regulatory landscape of melanoma reveals TEADS as regulators of the invasive cell state. Nat. Commun. 6, 16 (2015).

29. Cheng PF, Shakhova O, Widmer DS et al. Methylation-dependent SOX9 expression mediates invasion in human melanoma cells and is a negative prognostic factor in advanced melanoma. Genome Biol. 16(1), 42 (2015).

30. Hoek KS, Eichhoff OM, Schlegel NC et al. In vivo switching of human melanoma cells between proliferative and invasive states. Cancer Res. 68(3), 650-656 (2008).

31. Bolger AM, Lohse M, Usadel B. Trimmomatic: a flexible trimmer for Illumina sequence data. Bioinformatics 30(15), 2114-2120 (2014).

32. Kim D, Pertea G, Trapnell C, Pimentel H, Kelley R, Salzberg S. TopHat2: accurate alignment of transcriptomes in the presence of insertions, deletions and gene fusions. Genome Biol. 14(4), R36 (2013).

33. Anders S, Pyl PT, Huber W. HTSeq - a Python framework to work with high-throughput sequencing data. Bioinformatics 31(2), 166-169 (2015).

34. Love MI, Huber W, Anders S. Moderated estimation of fold change and dispersion for RNA-seq data with DESeq2. Genome Biol. 15(12), 1-21 (2014).

35. Derrien T, Johnson R, Bussotti G et al. The GENCODE v7 catalog of human long noncoding RNAs: analysis of their gene structure, evolution, and expression. Genome Res. 22(9), 1775-1789 (2012).

36. Trapnell C, Roberts A, Goff L et al. Differential gene and transcript expression analysis of RNA-seq experiments with TopHat and Cufflinks. Nat. Protoc. 7(3), 562-578 (2012).

37. Mi H, Huang X, Muruganujan A et al. PANTHER version 11: expanded annotation data from Gene Ontology and Reactome pathways, and data analysis tool enhancements. Nucleic Acids Res. 45(D1), D183-D189 (2017).

38. Chatterjee A, Rodger EJ, Stockwell PA, Weeks RJ, Morison IM. Technical considerations for reduced representation bisulfite sequencing with multiplexed libraries. BioMed Res. Int. 2012, 741542 (2012).

39. Stockwell PA, Chatterjee A, Rodger EJ, Morison IM. DMAP: differential methylation analysis package for RRBS and WGBS data. Bioinformatics 30(13), 1814-1822 (2014).

40. Krueger F, Andrews SR. Bismark: a flexible aligner and methylation caller for bisulfite-seq applications. Bioinformatics 27(11), 1571-1572 (2011).

41. Lee YM, Shin H, Choi W, Ahn S, Kim W. Characterization of human SMARCE1r high-mobility-group protein. Biochim. Biophys. Acta 1574(3), 269-276 (2002).

42. Liu Y, Sun J, Zhao M. ONGene: a literature-based database for human oncogenes. J. Genet. Genomics 44(2), 119-121 (2017). 
43. Zhao M, Kim P, Mitra R, Zhao J, Zhao Z. TSGene 2.0: an updated literature-based knowledgebase for tumor suppressor genes. Nucleic Acids Res. 44(D1), D1023-D1031 (2016).

44. Sondka Z, Bamford S, Cole CG, Ward SA, Dunham I, Forbes SA. The COSMIC Cancer Gene Census: describing genetic dysfunction across all human cancers. Nat. Rev. Cancer 18(11), 696-705 (2018).

45. Kulakovskiy IV, Vorontsov IE, Yevshin IS et al. HOCOMOCO: towards a complete collection of transcription factor binding models for human and mouse via large-scale ChIP-Seq analysis. Nucleic Acids Res. 46(D1), D252-D259 (2018).

46. Fornes O, Castro-Mondragon JA, Khan A et al. JASPAR 2020: update of the open-access database of transcription factor binding profiles. Nucleic Acids Res. 48(D1), D87-D92 (2019).

47. Kath R, Jambrosic JA, Holland L, Rodeck U, Herlyn M. Development of invasive and growth factor-independent cell variants from primary human melanomas. Cancer Res. 51(8), 2205-2211 (1991).

48. Hendrix MJC, Seftor EA, Meltzer PS et al. The stem cell plasticity of aggressive melanoma tumor cells. In: Stem Cells Handbook. Sell S (Ed.). Humana Press, NJ, USA, 297-306 (2004).

49. Reed JA, Finnerty B, Albino AP. Divergent cellular differentiation pathways during the invasive stage of cutaneous malignant melanoma progression. Am. J. Pathol. 155(2), 549-555 (1999).

50. Bröcker EB, Magiera H, Herlyn M. Nerve growth and expression of receptors for nerve growth factor in tumors of melanocyte origin. J. Invest. Dermatol. 96(5), 662-665 (1991).

51. Fang D, Hallman J, Sangha $\mathrm{N}$ et al. Expression of microtubule-associated protein 2 in benign and malignant melanocytes: implications for differentiation and progression of cutaneous melanoma. Am. J. Pathol. 158(6), 2107-2115 (2001).

52. Hoek KS, Schlegel NC, Brafford P et al. Metastatic potential of melanomas defined by specific gene expression profiles with no BRAF signature. Pigment Cell Melanoma Res. 19(4), 290-302 (2006).

53. Cheng W-Y, Kandel JJ, Yamashiro DJ, Canoll P, Anastassiou D. A multi-cancer mesenchymal transition gene expression signature is associated with prolonged time to recurrence in glioblastoma. PLoS One 7(4), e34705 (2012).

54. Kim H, Watkinson J, Varadan V, Anastassiou D. Multi-cancer computational analysis reveals invasion-associated variant of desmoplastic reaction involving INHBA, THBS2 and COL11A1. BMC Med. Genomics 3, 51 (2010).

55. Paluncic J, Kovacevic Z, Jansson PJ et al. Roads to melanoma: key pathways and emerging players in melanoma progression and oncogenic signaling. Biochim. Biophys. Acta Mol. Cell. Res. 1863(4), 770-784 (2016).

56. Richard G, Dalle S, Monet MA et al. ZEB1-mediated melanoma cell plasticity enhances resistance to MAPK inhibitors. EMBO Mol. Med. 8, 1143-1161 (2016).

57. Péterfi Z, Donkó Á, Orient A et al. Peroxidasin is secreted and incorporated into the extracellular matrix of myofibroblasts and fibrotic kidney. Am. J. Pathol. 175(2), 725-735 (2009).

58. Jayachandran A, Prithviraj P, Lo P-H et al. Identifying and targeting determinants of melanoma cellular invasion. Oncotarget 7(27), 41186-41202 (2016).

59. Ye IC, Fertig EJ, Digiacomo JW, Considine M, Godet I, Gilkes DM. Molecular portrait of hypoxia in breast cancer: a prognostic signature and novel HIF-regulated genes. Mol. Cancer Res. 16(10), 1889-1901 (2018).

60. Galante PA, Vidal DO, De Souza JE, Camargo AA, De Souza SJ. Sense-antisense pairs in mammals: functional and evolutionary considerations. Genome Biol. 8(3), R40 (2007).

61. Nie L, Wu HJ, Hsu JM et al. Long non-coding RNAs: versatile master regulators of gene expression and crucial players in cancer. Am. J. Transl. Res. 4(2), 127-150 (2012).

62. Muro EM, Andrade-Navarro MA. Pseudogenes as an alternative source of natural antisense transcripts. BMC Evol. Biol. 10, 338 (2010).

63. Frith MC, Wilming LG, Forrest A et al. Pseudo-messenger RNA: phantoms of the transcriptome. PLoS Genet. 2(4), e23 (2006).

64. Hu X, Yang L, Mo Y-Y. Role of pseudogenes in tumorigenesis. Cancers 10(8), 256 (2018).

65. Su Y, Vilgelm AE, Kelley MC et al. RAF265 inhibits the growth of advanced human melanoma tumors. Clin. Cancer Res. 18(8), 2184-2198 (2012).

66. Chen F, Zhang Y, Varambally S, Creighton CJ. Molecular correlates of metastasis by systematic pan-cancer analysis across The Cancer Genome Atlas. Mol. Cancer Res. 17(2), 476-487 (2019).

67. Sanchez E. Study of the cooperative effect of UVR, BRAF and LKB1 in melanoma (2019). www.tdx.cat/bitstream/handle/10803/667375/egs1de1.pdf?sequence=1

68. Sheahan AV, Morel KL, Burkhart DL et al. Targeting EZH2 increases therapeutic efficacy of check-point blockade in models of prostate cancer. BioRxiv doi:10.1101/730135 730135 (2019) (Epub ahead of print).

69. Chapman AR, Lee DF, Cai W et al. Correlated gene modules uncovered by single-cell transcriptomics with high detectability and accuracy. BioRxiv doi:10.1101/2019.12.31.892190 892190 (2020) (Epub ahead of print).

70. Wu Y-Y, Kuo H-C. Functional roles and networks of non-coding RNAs in the pathogenesis of neurodegenerative diseases. J. Biomed. Sci. 27(1), $49(2020)$. 
71. Xu TP, Liu XX, Xia R et al. SP1-induced upregulation of the long noncoding RNA TINCR regulates cell proliferation and apoptosis by affecting KLF2 mRNA stability in gastric cancer. Oncogene 34(45), 5648-5661 (2015).

72. Gong $\mathrm{H}$, Wen $\mathrm{H}$, Zhu X et al. High expression of long non-coding RNA ZEB1-AS1 promotes colorectal cancer cell proliferation partially by suppressing p15 expression. Tumour Biol. 39(6), 1010428317705336 (2017).

73. Liu M, Gou L, Xia J et al. lncRNA ITGB2-AS1 could promote the migration and invasion of breast cancer cells through up-regulating ITGB2. Int. J. Mol. Sci. 19(7), 1866 (2018).

74. Su H. ST, Wang H, Shi G, Zhang H, Sun F, Ye D. Decreased TCL6 expression is associated with poor prognosis in patients with clear cell renal cell carcinoma. Oncotarget 8, 5789-5799 (2016).

75. Zhang ZY, Lu YX, Zhang ZY et al. Loss of TINCR expression promotes proliferation, metastasis through activating EpCAM cleavage in colorectal cancer. Oncotarget 7(16), 22639-22649 (2016).

76. Zhang J, Li Z, Liu L et al. Long noncoding RNA TSLNC8 is a tumor suppressor that inactivates the interleukin-6/STAT3 signaling pathway. Hepatology 67(1), 171-187 (2018).

77. Zheng ZG, Xu H, Suo SS et al. The essential role of H19 contributing to cisplatin resistance by regulating glutathione metabolism in high-grade serous ovarian cancer. Sci. Rep. 6, 26093 (2016).

78. Huang Z, Lei W, Tan J, Hu HB. Long noncoding RNA LINC00961 inhibits cell proliferation and induces cell apoptosis in human non-small cell lung cancer. J. Cell Biochem. 119(11), 9072-9080 (2018).

79. Chen D, Zhu M, Su H, Chen J, Xu X, Cao C. LINC00961 restrains cancer progression via modulating epithelial-mesenchymal transition in renal cell carcinoma. J. Cell Physiol. 234(5), 7257-7265 (2019).

80. Robertson AG, Shih J, Yau C et al. Integrative analysis identifies four molecular and clinical subsets in uveal melanoma. Cancer Cell 32(2), 204-220.e215 (2017).

81. Lin X, Spindler TJ, De Souza Fonseca MA et al. Super-enhancer-associated lncRNA UCA1 interacts directly with AMOT to activate YAP target genes in epithelial ovarian cancer. iScience 17, 242-255 (2019).

82. Iyer MK, Niknafs YS, Malik R et al. The landscape of long noncoding RNAs in the human transcriptome. Nat. Genet. 47(3), 199-208 (2015).

83. Kaiser P, Seufert W, Höfferer L et al. A human ubiquitin-conjugating enzyme homologous to yeast UBC8. J. Biol. Chem. 269(12), 8797-8802 (1994).

84. Yim CY, Sekula DJ, Hever-Jardine MP et al. G0S2 suppresses oncogenic transformation by repressing a MYC-regulated transcriptional program. Cancer Res. 76(5), 1204-1213 (2016).

85. Hashimoto A, Hashimoto S, Sugino $\mathrm{H}$ et al. ZEB1 induces EPB41L5 in the cancer mesenchymal program that drives ARF6-based invasion, metastasis and drug resistance. Oncogenesis 5(9), e259 (2016).

86. Hosono T, Tanaka T, Tanji K, Nakatani T, Kamitani T. NUB1, an interferon-inducible protein, mediates anti-proliferative actions and apoptosis in renal cell carcinoma cells through cell-cycle regulation. Br. J. Cancer 102(5), 873-882 (2010).

87. Jern P, Coffin JM. Effects of retroviruses on host genome function. Annu. Rev. Genet. 42, 709-732 (2008).

88. Xie M, Hong C, Zhang B et al. DNA hypomethylation within specific transposable element families associates with tissue-specific enhancer landscape. Nat. Genet. 45(7), 836-841 (2013).

89. Du Q, Guo X, Zhang X et al. SYNJ2 variant rs 9365723 is associated with colorectal cancer risk in Chinese Han population. Int. J. Biol. Markers 31(2), e138-e143 (2016).

90. Ben-Chetrit N, Chetrit D, Russell R et al. Synaptojanin 2 is a druggable mediator of metastasis and the gene is overexpressed and amplified in breast cancer. Sci. Signal. 8(360), ra7 (2015).

91. Phillips JE, Corces VG. CTCF: master weaver of the genome. Cell 137(7), 1194-1211 (2009).

92. Wu J, Li L, Jiang G, Zhan H, Wang N. B-cell CLL/lymphoma 3 promotes glioma cell proliferation and inhibits apoptosis through the oncogenic STAT3 pathway. Int. J. Oncol. 49(6), 2471-2479 (2016).

93. Legge DN, Shephard AP, Collard TJ et al. BCL-3 promotes a cancer stem cell phenotype by enhancing $\beta$-catenin signalling in colorectal tumour cells. Dis. Model Mech. 12(3), (2019).

94. Prokhortchouk A, Hendrich B, Jørgensen $\mathrm{H}$ et al. The p120 catenin partner Kaiso is a DNA methylation-dependent transcriptional repressor. Genes Dev. 15(13), 1613-1618 (2001).

95. Pierre CC, Hercules SM, Yates C, Daniel JM. Dancing from bottoms up - roles of the POZ-ZF transcription factor Kaiso in cancer. Biochim. Biophys. Acta Rev. Cancer 1871(1), 64-74 (2019).

96. Kamimae S, Yamamoto E, Kai M et al. Epigenetic silencing of NTSR1 is associated with lateral and noninvasive growth of colorectal tumors. Oncotarget 6(30), 29975-29990 (2015).

97. Chang ZW, Dong L, Qin YR, Song M, Guo HY, Zhu QL. Correlations between gastric cancer family history and ROBO2 and RASSF2A gene methylations. J. Cancer Res. Ther. 12(2), 597-600 (2016). 
98. Lee M, Daniels MJ, Garnett MJ, Venkitaraman AR. A mitotic function for the high-mobility group protein HMG20b regulated by its interaction with the BRC repeats of the BRCA2 tumor suppressor. Oncogene 30(30), 3360-3369 (2011).

99. Rountree MR, Bachman KE, Baylin SB. DNMT1 binds HDAC2 and a new co-repressor, DMAP1, to form a complex at replication foci. Nat. Genet. 25(3), 269-277 (2000).

100. Ismail T, Lee H-K, Kim C, Kwon T, Park TJ, Lee H-S. KDM1A microenvironment, its oncogenic potential, and therapeutic significance. Epigenetics Chromatin 11(1), 33 (2018).

101. Seelig HP, Moosbrugger I, Ehrfeld H, Fink T, Renz M, Genth E. The major dermatomyositis-specific Mi-2 autoantigen is a presumed helicase involved in transcriptional activation. Arthritis Rheum. 38(10), 1389-1399 (1995).

102. Crawford NG, Kelly DE, Hansen MEB et al. Loci associated with skin pigmentation identified in African populations. Science 358(6365), eaan8433 (2017).

103. Blank JL, Liu XJ, Cosmopoulos K et al. Novel DNA damage checkpoints mediating cell death induced by the NEDD8-activating enzyme inhibitor MLN4924. Cancer Res. 73(1), 225-234 (2013).

104. Kiessling AA, Bletsa R, Desmarais B, Mara C, Kallianidis K, Loutradis D. Genome-wide microarray evidence that 8-cell human blastomeres over-express cell cycle drivers and under-express checkpoints. J. Assist. Reprod. Genet. 27(6), 265-276 (2010).

105. Thomas CP, Loftus RW, Liu KZ. AVP-induced VIT32 gene expression in collecting duct cells occurs via trans-activation of a CRE in the 5'-flanking region of the VIT32 gene. Am. J. Physiol. Renal Physiol. 287(3), F460-F468 (2004).

106. Nicod M, Michlig S, Flahaut $\mathrm{M}$ et al. A novel vasopressin-induced transcript promotes MAP kinase activation and ENaC downregulation. EMBO J. 21(19), 5109-5117 (2002). 